\title{
35. PALEOMAGNETISM OF DSDP LEG 55 BASALTS AND IMPLICATIONS FOR THE TECTONICS OF THE PACIFIC PLATE
}

\author{
Masaru Kono, Geophysical Institute, University of Tokyo, Tokyo 113, Japan
}

\section{INTRODUCTION}

The hot-spot origin of the Hawaiian and Emperor chains suggested by Wilson (1963a, b) and Morgan $(1971,1972)$ has become an attractive working hypothesis for the origin of these large volcanic features in the north-central Pacific Ocean. The age data gathered so far are in general agreement with movement of the Pacific plate over the hot spot at nearly constant speed of $8 \mathrm{~cm} /$ year (Dalrymple et al., this volume). Paleomagnetic data obtained at Midway Atoll (Grommé and Vine, 1972) and at Meiji Seamount at the northern end of the Emperor Seamount chain (Marshall, 1978) suggest low paleolatitudes for these volcanoes, and are therefore consistent with the hot-spot origin. Because the data are very limited, however, full evaluation of the hot-spot theory by paleomagnetic methods has been impossible to this day.

With the drilled samples from Ōjin (Hole 430A), Nintoku (Hole 432A), and Suiko seamounts (Holes 433A, 433B, 433C), we have tried to obtain reliable paleolatitudes of these seamounts and to reconstruct the movement of the Pacific plate in the period 0-70 m.y. with data from various sources. As the stability of natural remanent magnetization (NRM) is generally very high, and as we have samples from more than sixty separate lava flow units on Suiko (Hole 433C), we have enough material to assess the extent of paleosecular variation (PSV) in the North Pacific about 65 m.y. ago. As we shall see, the magnitude of PSV obtained from the present seamounts is much larger than the values reported for the recent and upper Tertiary volcanics on Hawaiian islands (Doell and Cox, 1971, 1972). This, in turn, suggests that the Midway and Meiji data may only represent partial sampling of PSV, and their paleolatitudes may contain much larger uncertainties than the nominal confidence intervals suggest.

\section{PALEOMAGNETIC DATA AND STABILITY OF REMANENCE}

Paleomagnetic measurements were carried out on more than 400 minicore samples, both on board the Glomar Challenger and in the paleomagnetic laboratory of the University of Tokyo. Sampling locations and other data are summarized in Table 1.

To obtain reliable paleomagnetic directions, all the samples were demagnetized, either by alternating field (AF) or by thermal methods. On board, samples were demagnetized by the AF method, using a Schonstedt GDS-1 demagnetizer, and measured using a Digico magnetometer. At least one (Flow Units 23-66 on Suiko) or two (Ōjin, Nintoku, and Flow Units $1-22$ on
Suiko) pilot sample(s) from each flow unit was (were) demagnetized and measured progressively at peak fields of $0,50,100,150,200,300,400$ and $500 \mathrm{Oe}$, and at 100 Oe steps thereafter until the median demagnetizing field $(\mathrm{MDF}=$ the peak AF at which half of the original remanence is demagnetized) or the highest possible field (1000 Oe) was reached. For the other samples, the NRM and the remanence after demagnetization at two or more peak fields, (the highest of which was 300 Oe or greater and also larger than the MDF of the sample) were measured. We employed such an exhaustive demagnetization procedure to ensure that the true paleomagnetic directions, and not some spurious effects due to defective experimental technique, were reached by these experiments. The MDF is a parameter which quite well characterizes the stability of remanence. Figure 1 shows that the amount of angular change in the direction of remanence during AF demagnetization is controlled by the MDF of the sample. A negative correlation (shown by the straight line) is apparent between the logarithms of these two parameters. Since the abscissa in this figure approximates the relative importance of the secondary components in the NRM, we see that the effect of secondary magnetization is unimportant in samples with MDFs of 150 Oe or higher; if the secondary magnetization is orthogonal to the primary NRM, it constitutes less than 10 per cent of the total NRM. Since most of the flow units have MDFs larger than this (Figure 2), their NRMs are essentially the primary magnetizations acquired when these rocks were formed, and are therefore good representatives of the ambient magnetic field of that time.

Figures 3 and 4 show typical examples of direction and intensity changes of remanence in progressive $\mathrm{AF}$ demagnetization. Since the MDF is a good parameter in classifying the stability of remanences (Figure 1 and 2), examples have been chosen so as to represent flow units with various MDFs. Figure 3 shows the least stable samples of the whole of Leg 55 (Site 433, Flow Unit 2) and another flow unit with a low mean MDF of $94 \mathrm{Oe}$ (Site 433, Flow Unit 10). They have NRMs which quickly decay in small alternating fields and show large changes in the direction of magnetization. Even in these samples, however, the direction of the primary component seems to be attained after demagnetization by 200 to $300 \mathrm{Oe}$; the dispersion in inclination is greatly reduced, and small change in magnetization takes place and the intensity decreases smoothly and slowly at higher demagnetizing fields. Figure 4 shows one of the flow units with a moderate MDF of 367 Oe (Site 432, Flow Unit 2) and one of the most stable flow units (Site 433, Flow Unit 14). In these examples, the intensity of 
TABLE 1

Paleomagnetic Samples of DSDP Leg 55

\begin{tabular}{|c|c|c|c|c|c|c|c|c|c|}
\hline \multirow[b]{2}{*}{ Seamount } & \multirow[b]{2}{*}{ Hole } & \multirow[b]{2}{*}{$\mathrm{Age}^{\mathrm{a}}$} & \multirow[b]{2}{*}{ Latitude } & \multirow[b]{2}{*}{ Longitude } & \multirow{2}{*}{$\begin{array}{l}\text { Water } \\
\text { Depth } \\
(\mathrm{m})\end{array}$} & \multirow{2}{*}{$\begin{array}{l}\text { Basement } \\
\text { Penetration } \\
\text { (m) }\end{array}$} & \multirow{2}{*}{$\begin{array}{l}\text { No. of } \\
\text { Flow Units }\end{array}$} & \multicolumn{2}{|c|}{ No. of Samples } \\
\hline & & & & & & & & On Board & On Shore \\
\hline$\overline{\mathrm{O}}$ jin & $430 \mathrm{~A}$ & $55.2 \pm 0.7$ & $37^{\circ} 59.3^{\prime} \mathrm{N}$ & $170^{\circ} 35.9^{\prime} \mathrm{E}$ & 1486 & 58.7 & $6(1)^{b}$ & $22(4)$ & 9 \\
\hline Nintoku & $432 \mathrm{~A}$ & $56.2 \pm 0.6$ & $41^{\circ} 20.0^{\prime} \mathrm{N}$ & $170^{\circ} 22.7^{\prime} \mathrm{E}$ & 1320 & 31.9 & $4(1)$ & $21(2)$ & $11(1)$ \\
\hline Suiko & $\begin{array}{l}433 \mathrm{~A} \\
433 \mathrm{~B} \\
433 \mathrm{C}\end{array}$ & $64.7 \pm 1.1$ & $\begin{array}{l}44^{\circ} 46.6^{\prime} \mathrm{N} \\
44^{\circ} 46.6^{\prime} \mathrm{N} \\
44^{\circ} 46.6^{\prime} \mathrm{N}\end{array}$ & $\begin{array}{l}170^{\circ} 01.3^{\prime} \mathrm{E} \\
170^{\circ} 01.2^{\prime} \mathrm{E} \\
170^{\circ} 01.2^{\prime} \mathrm{E}\end{array}$ & $\begin{array}{l}1874 \\
1874 \\
1874\end{array}$ & $\begin{array}{r}10.5 \\
23.0 \\
387.5\end{array}$ & $\begin{array}{r}1 \\
1 \\
65\end{array}$ & $\begin{array}{r}9 \\
4 \\
300\end{array}$ & $\begin{array}{r}3 \\
0 \\
73\end{array}$ \\
\hline
\end{tabular}

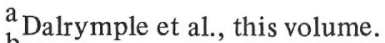

${ }^{\mathrm{N} u m b e r s}$ in parentheses indicate samples from volcaniclastic sandstones just above the basaltic basement.

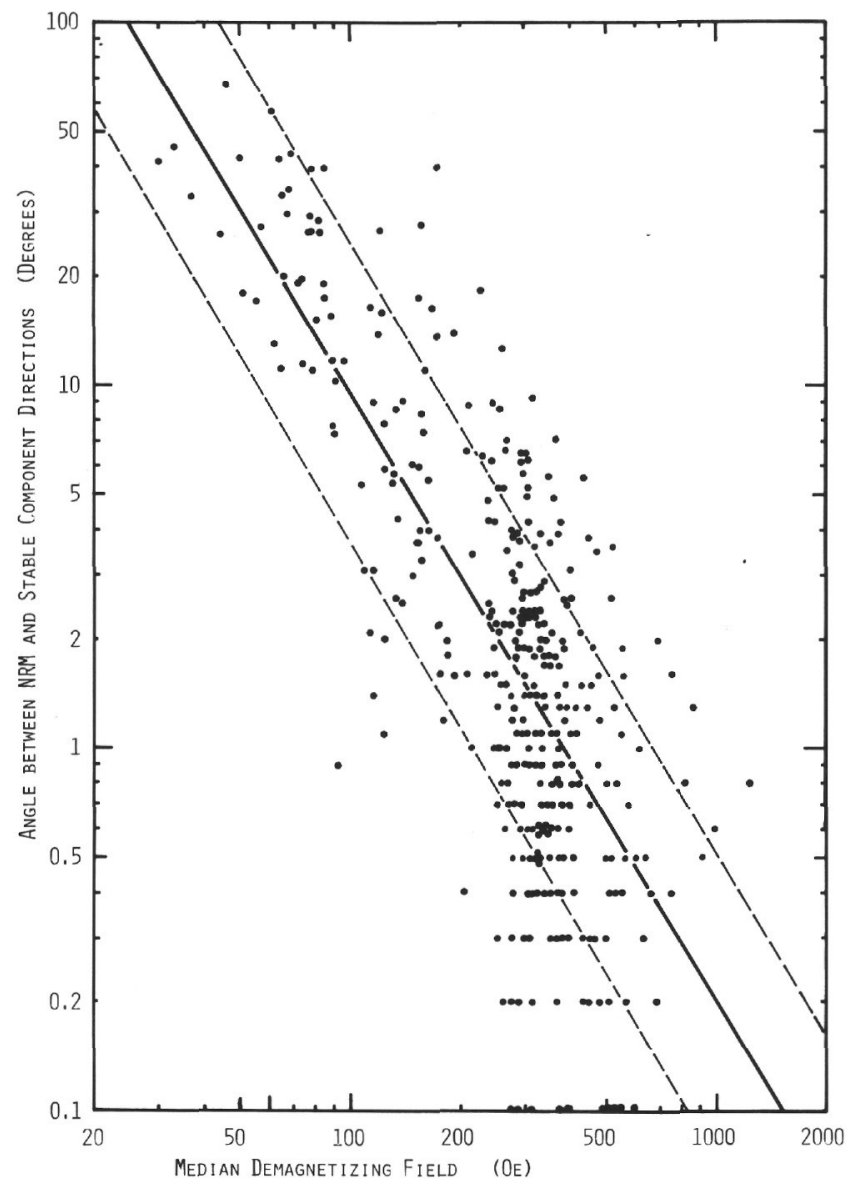

Figure 1. Relation between the MDF and the angular displacement of stable remanence from the NRM in individual samples. Angular displacement less than $0.1^{\circ}$ is set at $0.1^{\circ}$. The thick straight line and thin dashed lines indicate log-linear regression and $\pm 1 \sigma$ interval.

remanence decreases very smoothly and slowly while the direction of remanence changes very little in all the progressive AF steps. Figures 3 and 4 show the least stable, intermediate, and most stable samples of the Leg 55 basalts. As seen from Figure 2, the bulk of the flow units have mean MDFs of 300 Oe or higher and belong to the stable class. Even in the least stable samples, it is apparent that the primary component of magnetization can be separated by the application of AF demagnetization at a few hundred oersteds (Figure 3). We can therefore conclude that the direction of the primary magnetization is well kept in these rocks, and even in the least stable samples it can be easily determined by AF demagnetization (200-300 Oe). The original shipboard measurement data are tabulated in site reports for Sites 430,432 , and 433 (this volume).

Shore-based studies were carried out to supplement the on-board data. Magnetizations were measured using a Schonstedt spinner magnetometer. After measurement of the NRM, the samples were heated in air to $120^{\circ} \mathrm{C}$ and cooled in a nonmagnetic space of less than 100 gammas. Thermal demagnetization data are summarized in Table 2. For some of the samples, thermal demagnetization was continued to higher temperatures, in an effort to obtain estimates of paleointensities of the geomagnetic field (Kono and Tosha, this volume), but other samples were used for physical properties experiments (Kono, Hamano, and Morgan, this volume). After paleointensity experiments, we realized that for some samples $120^{\circ} \mathrm{C}$ was not enough to remove the secondary components; the samples with appreciable secondary components show direction changes not only between room temperature and $120^{\circ}$, but also at higher temperatures. Other samples were so stable that very little directional change occurred from room temperature to about $450^{\circ} \mathrm{C}$. Accordingly, we used data from thermally demagnetized samples (Table 2 ) in the calculation of flow mean directions if change was less than $5^{\circ}$ between NRM and demagnetized remanence, or when thermal demagnetization was carried out at higher temperatures and the direction of stable component was really reached.

The very high stability of remanence in Leg 55 basalts seems to be closely related to the mode of eruption of these rocks. Unlike the usual ocean flow basalts, these rocks are characterized by an almost universal occurrence of initial high-temperature oxidation (Kono, this volume; Kono, Clague, and Larson, this volume). As shown by optical and electron microscope observations, most of the titanomagnetites contain abundant ilmenite lamellae subdividing the host grain both at $\mu \mathrm{m}$ and sub- $\mu \mathrm{m}$ level (Kono, Clague, and Larson, this volume). Because of this subdivision, most of the grains seem to be in sin- 


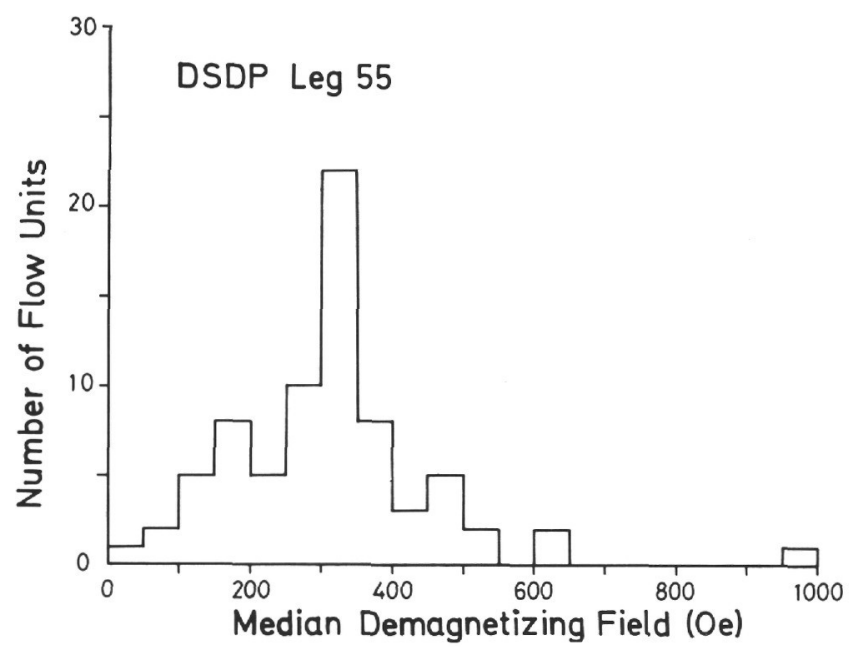

Figure 2. Histogram of flow mean MDFs of Leg $55 \mathrm{ba}$ salts and sandstones.

gle-domain or pseudo-single-domain range. They have large Königsberger ratios, small initial susceptibility, and high $J_{r} / J_{s}$ ratios, which all suggest a high magnetic stability (Kono, this volume). Some samples also show traces of low-temperature oxidation, but such secondary alteration is not widespread. Low-temperature oxidation seems to have occurred in a very limited place in lava flows. There is no systematic difference between the inclinations of high-temperature-oxidized and lowtemperature-oxidized samples from the same flow units. It seems, therefore, that even when low-temperature oxidation occurred it was not so severe as to disturb the direction of the original thermoremanent magnetization (TRM). In this sense, there is very little possibility that NRM in these rocks is affected by chemical remanent magnetization (CRM).

\section{FLOW MEAN INCLINATIONS}

Flow mean inclinations were calculated using both onboard data and data obtained on shore. Mean inclinations were calculated by a method similar to that of Briden and Ward (1966), assuming a Fisher distribution of magnetic directions (see Appendix, which follows this chapter). Stable component direction was taken at the AF demagnetization step where the error angle $\alpha_{95}$ is the smallest. Thermal demagnetization data were included in the final calculation when the data satisfied the conditions stated in the last section. Some of the samples taken from narrow zones below the flow unit boundaries showed inclinations very different from the mean inclinations of the flow units to which they belong but similar to the inclinations of the flows above the contacts. Such were interpreted as the result of baking of the uppermost parts of flow units by overlying lavas, and the data were excluded from the following analyses. Results are summarized in Table 3 and shown in Figures 5 and 6. The virtual geomagnetic latitude (VGL) in this table was calculated by the dipole formula
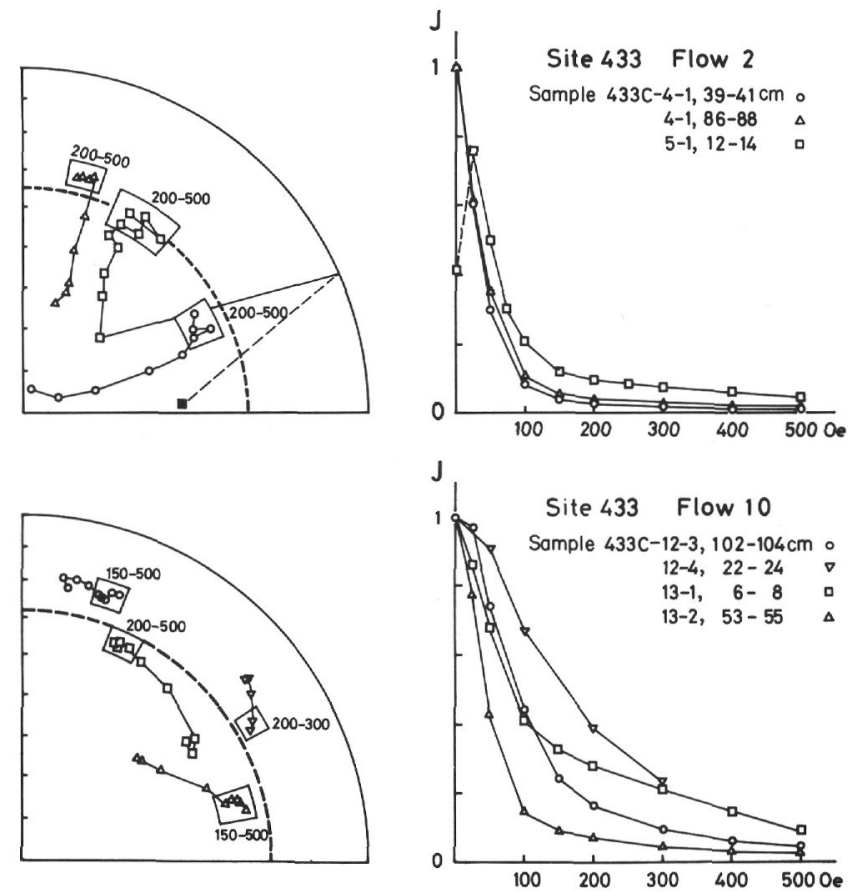

Figure 3. Examples of AF demagnetization of NRMs in typical flow units. Diagrams at right show the decay of remanence intensity of individual samples by AF demagnetization. Diagrams at left show changes in the direction of remanences with arbitrary declination. Thick dashed curves indicate flow mean inclinations (Table 3). At moderate to high AF (indicated by small numerals near boxes), the remanences attain directions which can be taken as those of primary magnetizations. All the directions except the NRM of Sample 433C-5-1, 12-14 cm (black square) are in the upper hemisphere of equal-area projection. The least stable flow unit of Leg 55 (Site 433, Flow 2) and a flow unit with a mean MDF of less than 100 Oe (Site 433, Flow 10).

$$
\tan \phi=\frac{1}{2} \tan I
$$

where $\phi$ is the VGL and $I$ the flow mean inclination.

Even after data of baked rocks were omitted, some of the flow units showed a large dispersion of inclinations. As the NRMs of most of the samples are quite stable, such large dispersions $\left(\alpha_{95} \geq 10^{\circ}\right)$ cannot be attributed to sampling errors, effects of secondary components, etc., when the number of samples is adequate $(N \geq 5)$. In fact, the grouping of the inclination data is usually so good that flow unit boundaries were detected at least at two places where onboard core descriptions failed to do so (flow boundaries $28 \mathrm{~A} / 28 \mathrm{~B}$ and $51 \mathrm{~A} / 51 \mathrm{~B}$ on Suiko). Some of the flow units are seen to contain several subunits, according to the initial description (e.g., Flow Units 4, 11, 19, etc. on Suiko). However, separate "magnetic units" were defined only when some systematic changes were observed across the sub-unit boundaries 

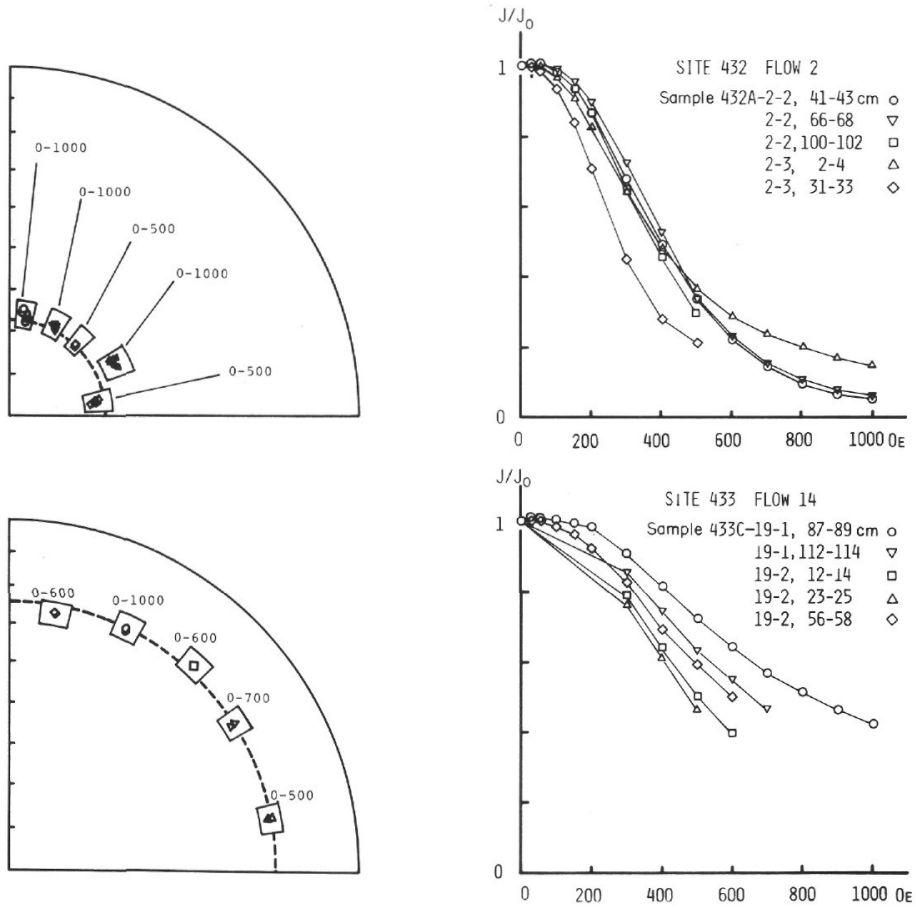

Figure 4. Examples of AF demagnetization of NRMs in typical flow units. Diagrams at right show the decay of remanence intensity of individual samples by AF demagnetization. Diagrams at left show changes in the direction of remanences with arbitrary declination. Thick dashed curves indicate flow mean inclinations (Table 3). At moderate to high AF (indicated by small numerals near boxes), the remanences attain directions which can be taken as those of primary magnetizations. All the directions except the NRM of Sample 433C-5-1, 12-14 cm (black square) are in the upper hemisphere of equal-area projection. Flow units with moderate or high MDFs. Site 432, Flow 2 (top) has an MDF of 367 Oe and Site 433, Flow 14 (bottom) has an MDF of 610 Oe.

(Flow Units 11A, 11B; 15A, 15B; 26A, 26B). After the subdivision of flow units described above, there remain only several units with large dispersions (Site 432, sandstone; Site 433, Flow Units 2, 10, 28A, and 51A). But all of them have only three or four samples, and the first three units have MDFs less than $100 \mathrm{Oe}$, so the large dispersions seem to result mainly from low stability of NRM and/or insufficient sampling.

Unlike sedimentary rocks, lava flows represent only spot samplings of geological time. Even if we have 65 lavas on Suiko, we do not know how many of them can be treated as really independent data. One way to assess this problem is to somehow detect time gaps in the stratigraphic sequence. In Figure 6, three sets of data which may indicate some time gaps are also shown. The first is the change in bulk chemistry (Kirkpatrick et al., this volume) which assumes that some time may have elapsed while the composition of the magma chamber changed in correspondence to different chemical groups. The second comes from descriptions of flow unit boundaries (site report for Site 433, this volume). Flow unit boundaries in this sequence of subaerially erupted lava flows are discernible by the occurrence of one or more of the following characteristic features: oxidation (red top), vesicularity, glass, brecciation, or erosion. Among these, oxidation and erosion of the surfaces of lavas cannot occur instantly, although it is difficult to estimate how many years are needed for these features to develop. The last estimate comes directly from the magnetic data. A jump in inclination of more than $15^{\circ}$ may be taken to represent a time gap of more than a few hundred years (assuming secular variation similar to that of recent times). Figure 6 indicates the possible time gaps estimated by petrological, geological, and magnetic data. As seen from the figure, the three methods suggest many probable time gaps in the lava flow sequence of Suiko. From this we may conclude that the number of independent samples in this sequence is not different from the number of flow units-within perhaps a factor of 2 .

The above considerations suggest that we may not be in great error if we assume each flow mean inclination to be independent. To be on the safe side, the following analysis was performed. For the adjacent two sample groups, a statistic $Z$ was calculated.

$$
Z=\frac{\bar{I}_{1}-\bar{I}_{2}}{\left(\frac{S_{1}^{2}}{n_{1}}-\frac{S_{2}^{2}}{n_{2}}\right)^{1 / 2}}
$$

where $\bar{I}, S$, and $n$ are the arithmetic mean and standard deviation of inclinations and the number of samples. If $Z$ is greater than 1.960 , we can decide that the difference between the two groups is real at the 5 per cent level of significance (Crow et al., 1960, p. 53). If the difference 
TABLE 2

Thermal Demagnetization Data of Leg 55 Basalts

\begin{tabular}{|c|c|c|c|c|c|c|c|c|c|c|c|c|}
\hline Hole & $\begin{array}{l}\text { Core- } \\
\text { Sec. }\end{array}$ & $\begin{array}{l}\text { Interval } \\
\text { in } \mathrm{cm}\end{array}$ & Flow & $I$ (NRM) & $D$ (NRM) & $J(\mathrm{NRM})$ & $I(120)$ & $D(120)$ & $J(120)$ & DIF & Ratio & Note \\
\hline $430 \mathrm{~A}$ & $5-2$ & 30 & 1 & -16.7 & 263.5 & $1.63 \mathrm{E}-03$ & -17.5 & 248.2 & $1.73 \mathrm{E}-03$ & 14.642 & 1.061 & $\mathrm{a}$ \\
\hline $430 \mathrm{~A}$ & $5-3$ & 107 & 1 & -42.6 & 120.5 & $1.07 \mathrm{E}-03$ & -33.6 & 140.5 & $1.21 \mathrm{E}-03$ & 18.064 & 1.131 & a \\
\hline $430 \mathrm{~A}$ & $5-5$ & 44 & 2 & -19.8 & 92.8 & $7.18 \mathrm{E}-03$ & -20.2 & 92.5 & $7.21 \mathrm{E}-03$ & 0.490 & 1.004 & \\
\hline $430 \mathrm{~A}$ & $5-5$ & 103 & 2 & -16.6 & 5.4 & $4.88 \mathrm{E}-03$ & -20.9 & 5.7 & $5.03 \mathrm{E}-03$ & 4.309 & 1.031 & \\
\hline $430 \mathrm{~A}$ & $6-1$ & 67 & 2 & -10.0 & 248.4 & $5.58 \mathrm{E}-03$ & -10.1 & 247.9 & $5.83 \mathrm{E}-03$ & 0.502 & 1.045 & \\
\hline $430 \mathrm{~A}$ & $6-1$ & 88 & 2 & -13.0 & 334.8 & $5.41 \mathrm{E}-03$ & -12.4 & 334.8 & $5.59 \mathrm{E}-03$ & 0.600 & 1.033 & \\
\hline $430 \mathrm{~A}$ & $6-2$ & 54 & 3 & -23.7 & 348.4 & $3.56 \mathrm{E}-03$ & -25.0 & 348.1 & $3.66 \mathrm{E}-03$ & 1.329 & 1.028 & \\
\hline $430 \mathrm{~A}$ & $6-2$ & 122 & 3 & -20.8 & 101.5 & $4.13 \mathrm{E}-03$ & -20.5 & 102.4 & $4.22 \mathrm{E}-03$ & 0.894 & 1.022 & \\
\hline $430 \mathrm{~A}$ & $6-4$ & 15 & 4 & -18.4 & 325.7 & $5.40 \mathrm{E}-03$ & -18.6 & 325.8 & $5.47 \mathrm{E}-03$ & 0.222 & 1.013 & \\
\hline $432 \mathrm{~A}$ & $2-1$ & 33 & SS & -54.8 & 332.6 & $3.48 \mathrm{E}-04$ & -40.1 & 291.9 & $1.73 \mathrm{E}-04$ & 30.614 & 0.497 & $\mathrm{a}$ \\
\hline $432 \mathrm{~A}$ & $2-1$ & 74 & 1 & -62.5 & 125.7 & 7.75E-03 & -65.0 & 121.9 & $7.72 \mathrm{E}-03$ & 3.011 & 0.996 & \\
\hline $432 \mathrm{~A}$ & $2-1$ & 107 & 1 & -63.7 & 211.1 & $5.15 \mathrm{E}-03$ & -63.9 & 207.8 & $5.25 \mathrm{E}-03$ & 1.471 & 1.019 & \\
\hline $432 \mathrm{~A}$ & $2-2$ & 72 & 2 & -68.7 & 306.6 & $8.82 \mathrm{E}-03$ & -66.0 & 304.8 & $9.35 \mathrm{E}-03$ & 2.787 & 1.060 & \\
\hline $432 \mathrm{~A}$ & $2-3$ & 12 & 2 & -62.6 & 247.0 & $2.82 \mathrm{E}-03$ & -63.1 & 245.7 & 2.93E-03 & 0.777 & 1.039 & \\
\hline $432 \mathrm{~A}$ & $2-3$ & 21 & 2 & -62.8 & 32.8 & $2.08 \mathrm{E}-03$ & -65.1 & 33.8 & $2.22 \mathrm{E}-03$ & 2.342 & 1.067 & \\
\hline $432 \mathrm{~A}$ & $3-2$ & 67 & 3 & -24.7 & 257.6 & $2.57 \mathrm{E}-03$ & -24.3 & 250.6 & $2.14 \mathrm{E}-03$ & 6.382 & 0.833 & a \\
\hline $432 \mathrm{~A}$ & $4-1$ & 64 & 3 & -31.9 & 144.6 & $7.22 \mathrm{E}-03$ & -30.0 & 146.2 & 7.22E-03 & 2.344 & 1.000 & \\
\hline $432 \mathrm{~A}$ & $4-1$ & 73 & 3 & -29.6 & 135.3 & 7.28E-03 & -29.0 & 136.1 & $7.36 \mathrm{E}-03$ & 0.921 & 1.011 & \\
\hline $432 \mathrm{~A}$ & $4-1$ & 120 & 3 & -28.3 & 128.0 & $3.87 \mathrm{E}-03$ & -28.2 & 126.4 & $3.85 \mathrm{E}-03$ & 1.413 & 0.995 & \\
\hline $432 \mathrm{~A}$ & $4-2$ & 80 & 3 & -27.4 & 141.6 & $8.36 \mathrm{E}-03$ & -29.3 & 140.7 & $8.22 \mathrm{E}-03$ & 2.059 & 0.983 & \\
\hline $432 \mathrm{~A}$ & $5-2$ & 69 & 3 & -44.8 & 192.4 & $2.53 \mathrm{E}-03$ & -31.6 & 196.2 & $1.97 \mathrm{E}-03$ & 13.529 & 0.779 & a \\
\hline $433 \mathrm{~A}$ & $20-2$ & 2 & 1 & -37.2 & 43.7 & $5.27 \mathrm{E}-03$ & -38.6 & 38.9 & $5.15 \mathrm{E}-03$ & 4.038 & 0.977 & \\
\hline $433 \mathrm{~A}$ & $20-2$ & 14 & 1 & -37.9 & 38.8 & $5.76 \mathrm{E}-03$ & -38.3 & 40.6 & $5.95 \mathrm{E}-03$ & 1.472 & 1.033 & \\
\hline $433 \mathrm{~A}$ & $20-2$ & 24 & 1 & -41.1 & 241.7 & $4.63 \mathrm{E}-03$ & -42.5 & 243.1 & $4.77 \mathrm{E}-03$ & 1.747 & 1.030 & \\
\hline $433 \mathrm{C}$ & $10-3$ & 92 & 4 & -40.5 & 174.6 & $1.80 \mathrm{E}-02$ & -40.9 & 174.0 & $1.89 \mathrm{E}-02$ & 0.607 & 1.050 & \\
\hline $433 C$ & $10-3$ & 145 & 4 & -38.7 & 148.0 & $1.85 \mathrm{E}-02$ & -38.6 & 148.8 & $1.84 \mathrm{E}-02$ & 0.634 & 0.995 & \\
\hline $433 \mathrm{C}$ & $10-4$ & 69 & 4 & -46.0 & 177.3 & $1.08 \mathrm{E}-02$ & -48.8 & 176.8 & $1.09 \mathrm{E}-02$ & 2.821 & 1.009 & \\
\hline $433 C$ & $10-6$ & 38 & 5 & -45.7 & 187.4 & $4.83 \mathrm{E}-03$ & -43.7 & 184.0 & 4. $26 \mathrm{E}-03$ & 3.137 & 0.882 & \\
\hline $433 \mathrm{C}$ & $11-1$ & 139 & 6 & -44.1 & 132.8 & $3.83 \mathrm{E}-03$ & -43.2 & 134.4 & $3.77 \mathrm{E}-03$ & 1.467 & 0.984 & \\
\hline $433 C$ & $11-3$ & 84 & 7 & -42.6 & 46.9 & $1.91 \mathrm{E}-03$ & -42.4 & 46.3 & $1.97 \mathrm{E}-03$ & 0.487 & 1.031 & \\
\hline $433 C$ & $11-3$ & 112 & 7 & -41.9 & 51.5 & $1.87 \mathrm{E}-03$ & -42.9 & 51.9 & $1.97 \mathrm{E}-03$ & 1.043 & 1.053 & \\
\hline $433 \mathrm{C}$ & $11-5$ & 36 & 8 & -54.0 & 120.3 & $5.43 \mathrm{E}-03$ & -73.6 & 53.0 & $3.37 \mathrm{E}-02$ & 32.845 & 6.206 & $\mathrm{a}$ \\
\hline $433 \mathrm{C}$ & $12-1$ & 109 & 9 & -60.0 & 259.9 & $2.84 \mathrm{E}-03$ & -60.4 & 263.0 & $2.90 \mathrm{E}-03$ & 1.592 & 1.021 & \\
\hline $433 C$ & $12-3$ & 5 & 9 & -43.1 & 271.9 & $1.67 \mathrm{E}-03$ & -42.2 & 263.9 & 1.79E-03 & 5.950 & 1.072 & $\mathrm{a}$ \\
\hline $433 C$ & $13-1$ & 125 & 10 & -64.9 & 301.7 & $2.30 \mathrm{E}-03$ & -62.1 & 297.7 & $1.69 \mathrm{E}-03$ & 3.319 & 0.735 & $\mathrm{a}$ \\
\hline $433 C$ & $13-2$ & 103 & 10 & -79.4 & 199.3 & $7.23 \mathrm{E}-04$ & -71.4 & 190.4 & $6.35 \mathrm{E}-04$ & 8.286 & 0.878 & \\
\hline $433 \mathrm{C}$ & 142 & 37 & $11 \mathrm{~A}$ & -32.9 & 177.1 & $2.66 \mathrm{E}-03$ & -32.6 & 177.4 & $2.66 \mathrm{E}-03$ & 0.394 & 1.000 & \\
\hline $433 \mathrm{C}$ & $15-1$ & 17 & $11 \mathrm{~B}$ & -57.9 & 168.9 & $2.68 \mathrm{E}-03$ & -54.2 & 170.1 & $2.73 \mathrm{E}-03$ & 3.760 & 1.019 & \\
\hline $433 \mathrm{C}$ & $15-4$ & 48 & 12 & -28.8 & 122.3 & $1.63 \mathrm{E}-03$ & -32.9 & 124.1 & $1.76 \mathrm{E}-03$ & 4.381 & 1.080 & $\mathrm{a}$ \\
\hline $433 \mathrm{C}$ & $15-4$ & 84 & 12 & -25.6 & 141.5 & $1.26 \mathrm{E}-03$ & -28.0 & 123.0 & $1.09 \mathrm{E}-03$ & 16.670 & 0.865 & a \\
\hline $433 \mathrm{C}$ & $15-5$ & 72 & 13 & -21.2 & 172.5 & $1.50 \mathrm{E}-03$ & -22.1 & 172.8 & $1.52 \mathrm{E}-03$ & 0.942 & 1.013 & \\
\hline $433 \mathrm{C}$ & $16-1$ & 80 & 13 & -55.5 & 340.9 & $3.24 \mathrm{E}-03$ & -46.6 & 336.7 & $2.28 \mathrm{E}-03$ & 9.279 & 0.704 & $\mathrm{a}$ \\
\hline $433 \mathrm{C}$ & $19-2$ & 9 & 14 & -25.8 & 137.8 & $9.49 \mathrm{E}-04$ & -24.6 & 137.7 & $9.24 \mathrm{E}-04$ & 1.204 & 0.974 & \\
\hline $433 \mathrm{C}$ & $19-2$ & 40 & 14 & -29.9 & 20.0 & $7.87 \mathrm{E}-04$ & -27.6 & 20.8 & 7.22E-04 & 2.405 & 0.917 & \\
\hline $433 C$ & $19-2$ & 64 & 14 & -25.3 & 10.2 & 4.29E-04 & -27.8 & 11.3 & $3.90 \mathrm{E}-04$ & 2.687 & 0.909 & \\
\hline $433 C$ & $19-3$ & 74 & $15 \mathrm{~A}$ & -35.9 & 3.2 & $2.48 \mathrm{E}-03$ & -38.6 & 3.8 & $2.57 \mathrm{E}-03$ & 2.742 & 1.036 & \\
\hline $433 \mathrm{C}$ & $20-1$ & 103 & $15 \mathrm{~B}$ & -61.3 & 142.3 & $5.56 \mathrm{E}-03$ & -59.5 & 145.6 & $5.62 \mathrm{E}-03$ & 2.428 & 1.011 & \\
\hline $433 \mathrm{C}$ & $20-2$ & 20 & $15 B$ & -58.3 & 246.1 & $5.41 \mathrm{E}-03$ & -30.3 & 209.8 & $5.78 \mathrm{E}-03$ & 37.354 & 1.068 & $\mathrm{a}$ \\
\hline $433 \mathrm{C}$ & $20-2$ & 35 & $15 \mathrm{~B}$ & -57.0 & 72.3 & $4.42 \mathrm{E}-03$ & -57.1 & 74.6 & $3.94 \mathrm{E}-03$ & 1.256 & 0.891 & \\
\hline $433 C$ & $21-1$ & 128 & 16 & -60.5 & 172.4 & $6.77 \mathrm{E}-03$ & -57.6 & 173.1 & $6.85 \mathrm{E}-03$ & 2.922 & 1.012 & \\
\hline $433 \mathrm{C}$ & $21-3$ & 7 & 17 & -63.5 & 162.5 & $2.54 \mathrm{E}-03$ & -61.0 & 164.5 & $2.54 \mathrm{E}-03$ & 2.668 & 1.000 & \\
\hline $433 \mathrm{C}$ & $21-4$ & 64 & 17 & -66.2 & 124.7 & $4.28 \mathrm{E}-03$ & -66.5 & 120.8 & $4.24 \mathrm{E}-03$ & 1.593 & 0.991 & \\
\hline $433 \mathrm{C}$ & $21-4$ & 101 & 17 & -52.1 & 334.5 & $3.97 \mathrm{E}-03$ & -55.8 & 332.0 & $3.77 \mathrm{E}-03$ & 3.981 & 0.950 & \\
\hline $433 \mathrm{C}$ & $22-1$ & 104 & 18 & -32.6 & 294.9 & $1.78 \mathrm{E}-03$ & -37.9 & 289.1 & $1.62 \mathrm{E}-03$ & 7.105 & 0.910 & $\mathrm{a}$ \\
\hline $433 \mathrm{C}$ & $23-4$ & 99 & 19 & -42.1 & 144.7 & $1.99 \mathrm{E}-03$ & -45.1 & 142.2 & $1.94 \mathrm{E}-03$ & 3.504 & 0.975 & \\
\hline $433 C$ & $25-2$ & 7 & 20 & -41.7 & 49.5 & $6.72 \mathrm{E}-04$ & -40.4 & 50.3 & $6.69 \mathrm{E}-04$ & 1.434 & 0.996 & \\
\hline $433 C$ & $25-2$ & 27 & 20 & -39.8 & 51.9 & $1.12 \mathrm{E}-03$ & -39.7 & 52.7 & $1.14 \mathrm{E}-03$ & 0.625 & 1.018 & \\
\hline $433 \mathrm{C}$ & $25-2$ & 54 & 20 & -39.6 & 242.3 & $1.50 \mathrm{E}-03$ & -38.2 & 238.4 & $1.46 \mathrm{E}-03$ & 3.342 & 0.973 & \\
\hline $433 \mathrm{C}$ & $25-5$ & 103 & 21 & -40.7 & 317.5 & $3.58 \mathrm{E}-03$ & -39.1 & 318.3 & $3.59 \mathrm{E}-03$ & 1.714 & 1.003 & \\
\hline $433 C$ & $26-5$ & 94 & 22 & -44.7 & 95.6 & $3.08 \mathrm{E}-03$ & -44.0 & 98.5 & $3.01 \mathrm{E}-03$ & 2.189 & 0.977 & \\
\hline $433 \mathrm{C}$ & $26-6$ & 129 & 23 & -41.4 & 177.4 & $1.79 \mathrm{E}-03$ & -42.6 & 176.2 & $1.81 \mathrm{E}-03$ & 1.496 & 1.011 & \\
\hline $433 \mathrm{C}$ & $27-5$ & 41 & 24 & -35.9 & 202.3 & $4.11 \mathrm{E}-04$ & -31.9 & 97.3 & $1.67 \mathrm{E}-03$ & 82.422 & 4.063 & $\mathrm{a}$ \\
\hline $433 \mathrm{C}$ & $28-4$ & 121 & $26 \mathrm{~A}$ & -69.1 & 59.5 & $1.47 \mathrm{E}-03$ & -69.3 & 62.3 & $1.53 \mathrm{E}-03$ & 1.014 & 1.041 & \\
\hline
\end{tabular}


TABLE 2 - Continued

\begin{tabular}{|c|c|c|c|c|c|c|c|c|c|c|c|c|}
\hline Hole & $\begin{array}{l}\text { Core- } \\
\text { Sec. }\end{array}$ & $\begin{array}{l}\text { Interval } \\
\text { in } \mathrm{cm}\end{array}$ & Flow & $I(\mathrm{NRM})$ & $D(\mathrm{NRM})$ & $J(\mathrm{NRM})$ & $I(120)$ & $D(120)$ & $J(120)$ & DIF & Ratio & Note \\
\hline $\begin{array}{l}433 C \\
433 C\end{array}$ & $\begin{array}{l}29-2 \\
31-4\end{array}$ & $\begin{array}{r}115 \\
83\end{array}$ & $\begin{array}{l}26 \mathrm{~B} \\
28 \mathrm{~A}\end{array}$ & $\begin{array}{l}-20.0 \\
-55.7\end{array}$ & $\begin{array}{r}272.8 \\
30.0\end{array}$ & $\begin{array}{l}2.56 \mathrm{E}-03 \\
1.18 \mathrm{E}-03\end{array}$ & $\begin{array}{r}25.9 \\
-56.3\end{array}$ & $\begin{array}{r}305.7 \\
29.6\end{array}$ & $\begin{array}{l}2.82 \mathrm{E}-03 \\
1.14 \mathrm{E}-03\end{array}$ & $\begin{array}{r}55.920 \\
0.642\end{array}$ & $\begin{array}{l}1.102 \\
0.966\end{array}$ & $\mathrm{a}$ \\
\hline $\begin{array}{l}433 C \\
433 C \\
433 C \\
433 C \\
433 C\end{array}$ & $\begin{array}{l}32-1 \\
35-1 \\
35-6 \\
35-6 \\
35-7\end{array}$ & $\begin{array}{r}38 \\
112 \\
48 \\
121 \\
116\end{array}$ & $\begin{array}{l}28 \mathrm{~B} \\
35 \\
35 \\
36 \\
36\end{array}$ & $\begin{array}{l}-44.6 \\
-48.1 \\
-47.4 \\
-50.2 \\
-52.8\end{array}$ & $\begin{array}{r}306.4 \\
201.0 \\
9.1 \\
349.6 \\
88.2\end{array}$ & $\begin{array}{l}2.43 \mathrm{E}-03 \\
3.29 \mathrm{E}-03 \\
7.86 \mathrm{E}-03 \\
2.57 \mathrm{E}-03 \\
1.65 \mathrm{E}-03\end{array}$ & $\begin{array}{l}-43.3 \\
-55.2 \\
-48.0 \\
-49.4 \\
-53.0\end{array}$ & $\begin{array}{r}308.8 \\
194.4 \\
9.6 \\
350.1 \\
87.6\end{array}$ & $\begin{array}{l}2.39 \mathrm{E}-03 \\
3.67 \mathrm{E}-03 \\
7.84 \mathrm{E}-03 \\
2.35 \mathrm{E}-03 \\
1.68 \mathrm{E}-03\end{array}$ & $\begin{array}{l}2.163 \\
8.188 \\
0.689 \\
0.863 \\
0.415\end{array}$ & $\begin{array}{l}0.984 \\
1.116 \\
0.997 \\
0.914 \\
1.018\end{array}$ & a \\
\hline $\begin{array}{l}433 C \\
433 C \\
433 C \\
433 C \\
433 C\end{array}$ & $\begin{array}{l}36-1 \\
36-1 \\
36-3 \\
36-4 \\
36-5\end{array}$ & $\begin{array}{r}45 \\
105 \\
60 \\
56 \\
29\end{array}$ & $\begin{array}{l}36 \\
37 \\
38 \\
39 \\
40\end{array}$ & $\begin{array}{l}-51.6 \\
-51.3 \\
-46.3 \\
-54.9 \\
-53.1\end{array}$ & $\begin{array}{r}140.8 \\
356.7 \\
261.0 \\
49.2 \\
329.1\end{array}$ & $\begin{array}{l}3.38 \mathrm{E}-03 \\
5.52 \mathrm{E}-03 \\
5.56 \mathrm{E}-03 \\
2.50 \mathrm{E}-03 \\
2.29 \mathrm{E}-03\end{array}$ & $\begin{array}{l}-51.9 \\
-51.7 \\
-45.5 \\
-57.9 \\
-47.5\end{array}$ & $\begin{array}{r}140.8 \\
357.1 \\
260.3 \\
53.6 \\
332.2\end{array}$ & $\begin{array}{l}3.14 \mathrm{E}-03 \\
5.10 \mathrm{E}-03 \\
5.15 \mathrm{E}-03 \\
2.36 \mathrm{E}-03 \\
2.15 \mathrm{E}-03\end{array}$ & $\begin{array}{l}0.302 \\
0.473 \\
0.938 \\
3.862 \\
5.938\end{array}$ & $\begin{array}{l}0.929 \\
0.924 \\
0.926 \\
0.944 \\
0.939\end{array}$ & $\mathrm{a}$ \\
\hline $\begin{array}{l}433 C \\
433 C \\
433 C \\
433 C \\
433 C\end{array}$ & $\begin{array}{l}37-3 \\
38-1 \\
38-5 \\
39-5 \\
39-6\end{array}$ & $\begin{array}{r}74 \\
57 \\
119 \\
96 \\
78\end{array}$ & $\begin{array}{l}44 \\
45 \\
47 \\
48 \\
48\end{array}$ & $\begin{array}{l}-54.9 \\
-46.9 \\
-18.4 \\
-42.8 \\
-47.6\end{array}$ & $\begin{array}{r}164.5 \\
56.9 \\
142.2 \\
92.4 \\
129.3\end{array}$ & $\begin{array}{l}3.68 \mathrm{E}-03 \\
2.22 \mathrm{E}-03 \\
9.75 \mathrm{E}-04 \\
1.08 \mathrm{E}-03 \\
1.30 \mathrm{E}-03\end{array}$ & $\begin{array}{l}-54.3 \\
-45.7 \\
-24.3 \\
-43.1 \\
-48.1\end{array}$ & $\begin{array}{r}165.0 \\
55.9 \\
140.6 \\
92.4 \\
128.9\end{array}$ & $\begin{array}{l}3.47 \mathrm{E}-03 \\
2.06 \mathrm{E}-03 \\
9.03 \mathrm{E}-04 \\
1.07 \mathrm{E}-03 \\
1.25 \mathrm{E}-03\end{array}$ & $\begin{array}{l}0.667 \\
1.386 \\
6.085 \\
0.302 \\
0.569\end{array}$ & $\begin{array}{l}0.943 \\
0.928 \\
0.926 \\
0.991 \\
0.962\end{array}$ & a \\
\hline $\begin{array}{l}433 C \\
433 C \\
433 C \\
433 C \\
433 C\end{array}$ & $\begin{array}{l}41-1 \\
42-1 \\
42-2 \\
42-5 \\
43-1\end{array}$ & $\begin{array}{r}51 \\
68 \\
136 \\
134 \\
33\end{array}$ & $\begin{array}{l}51 \mathrm{~B} \\
52 \\
53 \\
54 \\
54\end{array}$ & $\begin{array}{r}-9.9 \\
-14.5 \\
-25.8 \\
-36.7 \\
-37.6\end{array}$ & $\begin{array}{l}346.3 \\
174.3 \\
161.4 \\
200.2 \\
224.5\end{array}$ & $\begin{array}{l}4.22 \mathrm{E}-03 \\
3.26 \mathrm{E}-03 \\
2.11 \mathrm{E}-03 \\
4.63 \mathrm{E}-03 \\
3.64 \mathrm{E}-03\end{array}$ & $\begin{array}{l}-13.7 \\
-15.9 \\
-28.8 \\
-34.8 \\
-36.0\end{array}$ & $\begin{array}{l}346.5 \\
173.9 \\
161.0 \\
199.2 \\
222.8\end{array}$ & $\begin{array}{l}4.20 \mathrm{E}-03 \\
3.25 \mathrm{E}-03 \\
2.22 \mathrm{E}-03 \\
4.85 \mathrm{E}-03 \\
3.78 \mathrm{E}-03\end{array}$ & $\begin{array}{l}3.805 \\
1.452 \\
3.021 \\
2.066 \\
2.101\end{array}$ & $\begin{array}{l}0.995 \\
0.997 \\
1.052 \\
1.048 \\
1.038\end{array}$ & \\
\hline $\begin{array}{l}433 C \\
433 C \\
433 C \\
433 C \\
433 C\end{array}$ & $\begin{array}{l}44-1 \\
44-4 \\
45-2 \\
45-5 \\
45-6\end{array}$ & $\begin{array}{r}41 \\
108 \\
45 \\
51 \\
43\end{array}$ & $\begin{array}{l}56 \\
58 \\
59 \\
60 \\
60\end{array}$ & $\begin{array}{l}-49.8 \\
-45.3 \\
-46.8 \\
-54.5 \\
-49.5\end{array}$ & $\begin{array}{l}109.4 \\
345.8 \\
176.5 \\
270.7 \\
254.6\end{array}$ & $\begin{array}{l}2.82 \mathrm{E}-03 \\
6.30 \mathrm{E}-03 \\
5.78 \mathrm{E}-03 \\
4.10 \mathrm{E}-03 \\
2.37 \mathrm{E}-03\end{array}$ & $\begin{array}{l}-47.6 \\
-45.7 \\
-46.6 \\
-49.9 \\
-56.5\end{array}$ & $\begin{array}{l}110.2 \\
345.9 \\
177.6 \\
270.1 \\
250.6\end{array}$ & $\begin{array}{l}2.92 \mathrm{E}-03 \\
6.81 \mathrm{E}-03 \\
5.98 \mathrm{E}-03 \\
3.41 \mathrm{E}-03 \\
2.46 \mathrm{E}-03\end{array}$ & $\begin{array}{l}2.263 \\
0.409 \\
0.782 \\
4.615 \\
7.399\end{array}$ & $\begin{array}{l}1.035 \\
1.081 \\
1.035 \\
0.832 \\
1.038\end{array}$ & $a$ \\
\hline $\begin{array}{l}433 C \\
433 C \\
433 C \\
433 C \\
433 C \\
433 C\end{array}$ & $\begin{array}{l}46-3 \\
47-1 \\
47-5 \\
48-3 \\
49-1 \\
49-2\end{array}$ & $\begin{array}{r}54 \\
26 \\
25 \\
30 \\
142 \\
50\end{array}$ & $\begin{array}{l}62 \\
63 \\
64 \\
64 \\
66 \\
66\end{array}$ & $\begin{array}{l}-42.3 \\
-39.7 \\
-71.3 \\
-64.3 \\
-64.1 \\
-64.8\end{array}$ & $\begin{array}{r}314.2 \\
301.5 \\
282.7 \\
13.1 \\
324.9 \\
315.2\end{array}$ & $\begin{array}{l}3.49 \mathrm{E}-03 \\
3.13 \mathrm{E}-03 \\
1.04 \mathrm{E}-02 \\
5.72 \mathrm{E}-03 \\
3.06 \mathrm{E}-03 \\
4.29 \mathrm{E}-03\end{array}$ & $\begin{array}{l}-44.8 \\
-40.2 \\
-79.1 \\
-62.6 \\
-64.8 \\
-63.9\end{array}$ & $\begin{array}{r}314.5 \\
301.9 \\
295.3 \\
14.0 \\
324.7 \\
318.6\end{array}$ & $\begin{array}{l}3.36 \mathrm{E}-03 \\
3.31 \mathrm{E}-03 \\
3.46 \mathrm{E}-03 \\
5.85 \mathrm{E}-03 \\
3.21 \mathrm{E}-03 \\
4.48 \mathrm{E}-03\end{array}$ & $\begin{array}{l}2.510 \\
0.588 \\
8.394 \\
1.747 \\
0.706 \\
1.725\end{array}$ & $\begin{array}{l}0.963 \\
1.058 \\
0.333 \\
1.023 \\
1.049 \\
1.044\end{array}$ & $\mathrm{a}$ \\
\hline
\end{tabular}

${ }^{\mathrm{a}}$ Excluded from calculation of flow mean inclination.

was not significant, the two groups were combined into one and the comparison was made between this new group and the next (flow). In this way, some of the flow units were combined to form groups, while other flow units were determined to be independent. The groups thus formed are indicated in Figures 5 and 6 by boxes and the group inclination data are summarized in Table 4. It should be noted that there are about 40 independent inclination data for Suiko Seamount, even after the grouping.

\section{PALEOSECULAR VARIATION AND ESTIMATION OF PALEOLATITUDE}

One of the major objectives of DSDP Leg 55 was to determine paleolatitudes of the Emperor Seamounts by the paleomagnetic method. To do this it is essential to average out the fluctuations in inclination caused by paleosecular variation. We have seen that there are at least about 40 independent inclination data on Suiko. Now we show that they are really enough to characterize the paleosecular variation (PSV) about $65 \mathrm{~m} . \mathrm{y}$. ago in the north central Pacific.

In Figure 6, we see that some of the consecutive flow units have very similar inclinations; e.g., Flow Units 4 to 7 and 18 to 20 . This may indicate that these flow units erupted successively within a short time, say, a few hundred years. At other places, such as between Flow Units 27 and 45 and between 51A and 63, the inclination data define smooth curves against depth. This certainly reflects the change in the earth's magnetic field, i.e., secular variation. If the characteristic time scales of secular variation at the time of Suiko were similar to the ones in Tertiary and Recent times, such curves may represent a time interval between several hundred years and a few thousand years. There are also jumps in inclination trends at many places. These may correspond to time gaps of any length, but they are certainly longer than several hundred years. About 12 such inclination jumps can be seen in Figure 6. On the basis of rough estimates of time, discussed earlier, we can conclude that the flow units sampled on Suiko cover a time span of at least $10^{4}$ years, probably considerably longer than that.

On the other hand, all the flow units are reversely magnetized. According to the time scale of Heirtzler et al. (1968), the mean duration of a polarity interval was about $0.9 \mathrm{~m} . \mathrm{y}$. in the period of 45 to $80 \mathrm{~m} . \mathrm{y}$. ago. Since their time scale may neglect polarity intervals of short time duration, the true mean of a polarity interval may be even shorter. In fact, Blakely and Cox (1972) ana- 
TABLE 3

Flow Mean Inclinations, Latitudes, and Other Data

\begin{tabular}{|c|c|c|c|c|c|c|c|c|c|c|c|c|c|c|c|}
\hline Hole & Flow & $\begin{array}{l}\text { Top } \\
\text { (m) }\end{array}$ & $\begin{array}{l}\text { Bottom } \\
\text { (m) }\end{array}$ & $N$ & $(T)$ & $\mathrm{AF}$ & $I$ & VGL & $k$ & $R$ & $\alpha 95$ & $\bar{I}$ & $s_{1}$ & $\begin{array}{l}\text { MDF } \\
(\mathrm{Oe})\end{array}$ & $S_{M D F}$ \\
\hline \multicolumn{16}{|c|}{$\bar{O}$ jin } \\
\hline $\begin{array}{l}430 \mathrm{~A} \\
430 \mathrm{~A} \\
430 \mathrm{~A} \\
430 \mathrm{~A} \\
430 \mathrm{~A} \\
430 \mathrm{~A}\end{array}$ & $\begin{array}{l}\text { SS } \\
1 \\
2 \\
3 \\
4 \\
5\end{array}$ & $\begin{array}{l}57.0 \\
52.0 \\
71.4 \\
76.9 \\
79.2 \\
80.5\end{array}$ & $\begin{array}{l}52.5 \\
71.4 \\
76.9 \\
79.2 \\
80.6 \\
80.9\end{array}$ & $\begin{array}{l}4 \\
8 \\
8 \\
6 \\
2 \\
1\end{array}$ & $\begin{array}{l}(0) \\
(0) \\
(4) \\
(2) \\
(1) \\
(0)\end{array}$ & $\begin{array}{l}300 \\
200 \\
300 \\
300 \\
300 \\
300\end{array}$ & $\begin{array}{r}-43.2 \\
-18.1 \\
-17.4 \\
-22.4 \\
-18.8 \\
59.0\end{array}$ & $\begin{array}{r}25.2 \\
9.3 \\
8.9 \\
11.7 \\
9.6 \\
39.8\end{array}$ & $\begin{array}{r}4852.9 \\
313.3 \\
132.8 \\
583.3\end{array}$ & $\begin{array}{l}3.9994 \\
7.9777 \\
7.9473 \\
5.9914\end{array}$ & $\begin{array}{l}1.3 \\
3.1 \\
4.8 \\
2.8\end{array}$ & $\begin{array}{r}-43.2 \\
-18.1 \\
-17.3 \\
-22.4 \\
-18.8 \\
59.0\end{array}$ & $\begin{array}{l}0.9 \\
3.5 \\
5.3 \\
2.6 \\
0.2\end{array}$ & $\begin{array}{l}153 \\
212 \\
305 \\
323 \\
334 \\
116\end{array}$ & $\begin{array}{r}11 \\
105 \\
15 \\
26\end{array}$ \\
\hline \multicolumn{16}{|c|}{ Nintoku } \\
\hline $\begin{array}{l}432 \mathrm{~A} \\
432 \mathrm{~A} \\
432 \mathrm{~A} \\
432 \mathrm{~A}\end{array}$ & $\begin{array}{c}\text { SS } \\
1 \\
2 \\
3\end{array}$ & $\begin{array}{l}41.5 \\
42.0 \\
42.8 \\
55.0\end{array}$ & $\begin{array}{l}42.0 \\
42.8 \\
44.6 \\
73.8\end{array}$ & $\begin{array}{r}3 \\
5 \\
8 \\
14\end{array}$ & $\begin{array}{l}(1) \\
(2) \\
(3) \\
(4)\end{array}$ & $\begin{array}{l}300 \\
400 \\
300 \\
200\end{array}$ & $\begin{array}{l}-44.6 \\
-65.2 \\
-66.4 \\
-28.6\end{array}$ & $\begin{array}{l}26.3 \\
47.3 \\
48.9 \\
15.2\end{array}$ & $\begin{array}{r}263.6 \\
2200.1 \\
646.0 \\
1462.0\end{array}$ & $\begin{array}{r}2.9924 \\
4.9982 \\
7.9892 \\
13.9911\end{array}$ & $\begin{array}{l}7.6 \\
1.6 \\
2.2 \\
1.0\end{array}$ & $\begin{array}{l}-44.5 \\
-65.2 \\
-66.3 \\
-28.6\end{array}$ & $\begin{array}{l}4.3 \\
1.4 \\
2.4 \\
1.5\end{array}$ & $\begin{array}{l}121 \\
344 \\
367 \\
165\end{array}$ & $\begin{array}{r}3 \\
17 \\
52 \\
82\end{array}$ \\
\hline \multicolumn{16}{|c|}{ Suiko } \\
\hline $\begin{array}{l}433 A \\
433 C \\
433 C \\
433 C \\
433 C\end{array}$ & $\begin{array}{l}1 \\
2 \\
4 \\
5 \\
6\end{array}$ & $\begin{array}{l}163.5 \\
181.5 \\
202.7 \\
210.0 \\
214.2\end{array}$ & $\begin{array}{l}172.5 \\
192.0 \\
210.0 \\
214.2 \\
215.3\end{array}$ & $\begin{array}{r}18 \\
3 \\
17 \\
5 \\
4\end{array}$ & $\begin{array}{l}(3) \\
(0) \\
(4) \\
(1) \\
(1)\end{array}$ & $\begin{array}{l}300 \\
300 \\
200 \\
300 \\
200\end{array}$ & $\begin{array}{l}-39.0 \\
-34.7 \\
-43.0 \\
-44.1 \\
-43.0\end{array}$ & $\begin{array}{l}22.0 \\
19.1 \\
25.0 \\
25.9 \\
25.0\end{array}$ & $\begin{array}{r}414.1 \\
162.3 \\
233.1 \\
254.4 \\
1253.9\end{array}$ & $\begin{array}{r}17.9589 \\
2.9877 \\
16.9314 \\
4.9843 \\
3.9976\end{array}$ & $\begin{array}{l}1.7 \\
9.7 \\
2.3 \\
4.8 \\
2.6\end{array}$ & $\begin{array}{l}-38.9 \\
-34.6 \\
-42.9 \\
-44.0 \\
-43.0\end{array}$ & $\begin{array}{l}2.9 \\
5.6 \\
3.9 \\
3.9 \\
1.9\end{array}$ & $\begin{array}{r}98 \\
41 \\
275 \\
312 \\
311\end{array}$ & $\begin{array}{l}75 \\
11 \\
27 \\
22 \\
43\end{array}$ \\
\hline $\begin{array}{l}433 C \\
433 C \\
433 C \\
433 C \\
433 C\end{array}$ & $\begin{array}{c}7 \\
8 \\
9 \\
10 \\
11 \mathrm{~A}\end{array}$ & $\begin{array}{l}215.3 \\
217.9 \\
223.9 \\
226.8 \\
233.0\end{array}$ & $\begin{array}{l}217.9 \\
223.9 \\
226.8 \\
233.4 \\
235.9\end{array}$ & $\begin{array}{l}5 \\
4 \\
5 \\
4 \\
3\end{array}$ & $\begin{array}{l}(2) \\
(0) \\
(1) \\
(0) \\
(1)\end{array}$ & $\begin{array}{l}400 \\
200 \\
300 \\
300 \\
200\end{array}$ & $\begin{array}{l}-44.3 \\
-53.0 \\
-57.3 \\
-28.5 \\
-33.4\end{array}$ & $\begin{array}{l}26.0 \\
33.6 \\
37.9 \\
15.2 \\
18.2\end{array}$ & $\begin{array}{r}1671.2 \\
883.8 \\
1297.7 \\
154.3 \\
1065.5\end{array}$ & $\begin{array}{l}4.9976 \\
3.9966 \\
4.9969 \\
3.9806 \\
2.9981\end{array}$ & $\begin{array}{l}1.9 \\
3.1 \\
2.1 \\
7.4 \\
3.8\end{array}$ & $\begin{array}{l}-44.3 \\
-53.0 \\
-57.2 \\
-28.4 \\
-33.4\end{array}$ & $\begin{array}{l}1.6 \\
2.2 \\
1.8 \\
5.3 \\
2.2\end{array}$ & $\begin{array}{r}344 \\
335 \\
183 \\
94 \\
470\end{array}$ & $\begin{array}{r}21 \\
174 \\
62 \\
48 \\
139\end{array}$ \\
\hline $\begin{array}{l}433 C \\
433 C \\
433 C \\
433 C \\
433 C\end{array}$ & $\begin{array}{l}11 \mathrm{~B} \\
12 \\
13 \\
14 \\
15 \mathrm{~A}\end{array}$ & $\begin{array}{l}235.9 \\
245.6 \\
247.5 \\
262.0 \\
264.1\end{array}$ & $\begin{array}{l}245.7 \\
247.5 \\
262.0 \\
264.1 \\
270.0\end{array}$ & $\begin{array}{l}6 \\
6 \\
7 \\
8 \\
6\end{array}$ & $\begin{array}{l}(1) \\
(0) \\
(1) \\
(3) \\
(1)\end{array}$ & $\begin{array}{l}400 \\
300 \\
300 \\
300 \\
300\end{array}$ & $\begin{array}{l}-52.0 \\
-19.5 \\
-21.9 \\
-25.3 \\
-44.3\end{array}$ & $\begin{array}{l}32.6 \\
10.0 \\
11.4 \\
13.3 \\
26.0\end{array}$ & $\begin{array}{r}684.3 \\
783.2 \\
774.6 \\
1081.6 \\
184.3\end{array}$ & $\begin{array}{l}5.9927 \\
5.9936 \\
6.9923 \\
7.9935 \\
5.9729\end{array}$ & $\begin{array}{l}2.6 \\
2.4 \\
2.2 \\
1.7 \\
4.9\end{array}$ & $\begin{array}{l}-52.0 \\
-19.5 \\
-21.9 \\
-25.3 \\
-44.1\end{array}$ & $\begin{array}{l}2.3 \\
2.3 \\
2.2 \\
1.9 \\
4.6\end{array}$ & $\begin{array}{l}222 \\
329 \\
148 \\
610 \\
351\end{array}$ & $\begin{array}{r}129 \\
62 \\
154 \\
141 \\
250\end{array}$ \\
\hline $\begin{array}{l}433 C \\
433 C \\
433 C \\
433 C \\
433 C\end{array}$ & $\begin{array}{l}15 \mathrm{~B} \\
16 \\
17 \\
18 \\
19\end{array}$ & $\begin{array}{l}269.9 \\
271.4 \\
280.9 \\
289.0 \\
298.0\end{array}$ & $\begin{array}{l}271.4 \\
280.7 \\
289.0 \\
294.6 \\
317.7\end{array}$ & $\begin{array}{l}4 \\
5 \\
8 \\
6 \\
8\end{array}$ & $\begin{array}{l}(2) \\
(1) \\
(3) \\
(0) \\
(1)\end{array}$ & $\begin{array}{l}200 \\
300 \\
200 \\
200 \\
300\end{array}$ & $\begin{array}{l}-58.5 \\
-58.3 \\
-61.2 \\
-41.5 \\
-39.2\end{array}$ & $\begin{array}{l}39.2 \\
39.0 \\
42.3 \\
23.9 \\
22.1\end{array}$ & $\begin{array}{r}2584.0 \\
2105.7 \\
369.6 \\
1468.4 \\
303.9\end{array}$ & $\begin{array}{l}3.9988 \\
4.9981 \\
7.9811 \\
5.9966 \\
7.9770\end{array}$ & $\begin{array}{l}1.8 \\
1.7 \\
2.9 \\
1.7 \\
3.2\end{array}$ & $\begin{array}{l}-58.5 \\
-58.3 \\
-61.1 \\
-41.5 \\
-39.1\end{array}$ & $\begin{array}{l}1.3 \\
1.4 \\
3.2 \\
1.6 \\
3.5\end{array}$ & $\begin{array}{l}165 \\
198 \\
254 \\
152 \\
333\end{array}$ & $\begin{array}{r}11 \\
71 \\
109 \\
86 \\
77\end{array}$ \\
\hline $\begin{array}{l}433 C \\
433 C \\
433 C \\
433 C \\
433 C\end{array}$ & $\begin{array}{l}20 \\
21 \\
22 \\
23 \\
24\end{array}$ & $\begin{array}{l}317.6 \\
319.6 \\
330.2 \\
333.5 \\
336.1\end{array}$ & $\begin{array}{l}319.6 \\
330.2 \\
333.5 \\
335.0 \\
343.1\end{array}$ & $\begin{array}{l}8 \\
6 \\
5 \\
3 \\
9\end{array}$ & $\begin{array}{l}(3) \\
(1) \\
(1) \\
(1) \\
(0)\end{array}$ & $\begin{array}{l}200 \\
200 \\
600 \\
300 \\
200\end{array}$ & $\begin{array}{l}-40.1 \\
-38.1 \\
-43.0 \\
-41.5 \\
-36.2\end{array}$ & $\begin{array}{l}22.8 \\
21.4 \\
25.0 \\
23.9 \\
20.1\end{array}$ & $\begin{array}{r}2725.1 \\
889.2 \\
6170.0 \\
2439.7 \\
382.3\end{array}$ & $\begin{array}{l}7.9974 \\
5.9944 \\
4.9994 \\
2.9992 \\
8.9791\end{array}$ & $\begin{array}{l}1.1 \\
2.2 \\
1.0 \\
2.5 \\
2.6\end{array}$ & $\begin{array}{l}-40.1 \\
-38.1 \\
-43.0 \\
-41.5 \\
-36.1\end{array}$ & $\begin{array}{l}1.2 \\
2.1 \\
0.8 \\
1.4 \\
3.1\end{array}$ & $\begin{array}{l}539 \\
313 \\
633 \\
262 \\
211\end{array}$ & $\begin{array}{r}190 \\
77 \\
186 \\
115 \\
97\end{array}$ \\
\hline $\begin{array}{l}433 C \\
433 C \\
433 C \\
433 C \\
433 C\end{array}$ & $\begin{array}{l}25 \\
26 \mathrm{~A} \\
26 \mathrm{~B} \\
27 \\
28 \mathrm{~A}\end{array}$ & $\begin{array}{l}343.1 \\
350.2 \\
356.6 \\
357.9 \\
375.9\end{array}$ & $\begin{array}{l}350.3 \\
356.6 \\
357.9 \\
375.9 \\
378.7\end{array}$ & $\begin{array}{l}4 \\
5 \\
4 \\
5 \\
4\end{array}$ & $\begin{array}{l}(0) \\
(1) \\
(0) \\
(0) \\
(1)\end{array}$ & $\begin{array}{l}300 \\
200 \\
300 \\
200 \\
200\end{array}$ & $\begin{array}{l}-68.5 \\
-68.9 \\
-21.0 \\
-62.5 \\
-57.0\end{array}$ & $\begin{array}{l}51.8 \\
52.3 \\
10.9 \\
43.8 \\
37.6\end{array}$ & $\begin{array}{r}379.9 \\
6858.3 \\
832.5 \\
949.8 \\
149.1\end{array}$ & $\begin{array}{l}3.9921 \\
4.9994 \\
3.9964 \\
4.9958 \\
3.9799\end{array}$ & $\begin{array}{l}4.7 \\
0.9 \\
3.2 \\
2.5 \\
7.5\end{array}$ & $\begin{array}{l}-68.3 \\
-68.9 \\
-21.0 \\
-62.4 \\
-56.7\end{array}$ & $\begin{array}{l}3.2 \\
0.8 \\
2.3 \\
2.1 \\
5.8\end{array}$ & $\begin{array}{l}127 \\
253 \\
322 \\
114 \\
367\end{array}$ & $\begin{array}{r}71 \\
181 \\
51 \\
92 \\
76\end{array}$ \\
\hline $\begin{array}{l}433 C \\
433 C \\
433 C \\
433 C \\
433 C\end{array}$ & $\begin{array}{l}28 \mathrm{~B} \\
29 \\
30 \\
31 \\
32\end{array}$ & $\begin{array}{l}378.7 \\
387.5 \\
389.9 \\
394.7 \\
397.3\end{array}$ & $\begin{array}{l}387.5 \\
389.9 \\
394.7 \\
397.3 \\
403.6\end{array}$ & $\begin{array}{l}5 \\
5 \\
5 \\
4 \\
5\end{array}$ & $\begin{array}{l}(2) \\
(0) \\
(0) \\
(0) \\
(0)\end{array}$ & $\begin{array}{l}300 \\
200 \\
200 \\
200 \\
200\end{array}$ & $\begin{array}{l}-43.0 \\
-40.4 \\
-42.3 \\
-40.8 \\
-40.3\end{array}$ & $\begin{array}{l}25.0 \\
23.1 \\
24.4 \\
23.3 \\
23.0\end{array}$ & $\begin{array}{r}1622.6 \\
2627.2 \\
4314.2 \\
11693.5 \\
818.4\end{array}$ & $\begin{array}{l}4.9975 \\
4.9985 \\
4.9991 \\
3.9997 \\
4.9951\end{array}$ & $\begin{array}{l}1.9 \\
1.5 \\
1.2 \\
0.8 \\
2.7\end{array}$ & $\begin{array}{l}-43.0 \\
-40.4 \\
-42.3 \\
-40.8 \\
-40.3\end{array}$ & $\begin{array}{l}1.6 \\
1.3 \\
1.0 \\
0.6 \\
2.3\end{array}$ & $\begin{array}{l}340 \\
300 \\
385 \\
459 \\
339\end{array}$ & $\begin{array}{r}84 \\
24 \\
82 \\
130 \\
30\end{array}$ \\
\hline $\begin{array}{l}443 C \\
433 C \\
433 C \\
433 C \\
433 C\end{array}$ & $\begin{array}{l}33 \\
34 \\
35 \\
36 \\
37\end{array}$ & $\begin{array}{l}403.6 \\
406.6 \\
410.1 \\
420.4 \\
422.3\end{array}$ & $\begin{array}{l}406.6 \\
410.1 \\
420.4 \\
422.3 \\
423.5\end{array}$ & $\begin{array}{l}5 \\
5 \\
6 \\
7 \\
4\end{array}$ & $\begin{array}{l}(0) \\
(0) \\
(1) \\
(3) \\
(1)\end{array}$ & $\begin{array}{l}200 \\
200 \\
200 \\
500 \\
200\end{array}$ & $\begin{array}{l}-42.3 \\
-41.4 \\
-49.6 \\
-50.9 \\
-51.3\end{array}$ & $\begin{array}{l}24.5 \\
23.8 \\
30.4 \\
31.6 \\
31.9\end{array}$ & $\begin{array}{r}19446.9 \\
2316.9 \\
4224.3 \\
1227.6 \\
39130.0\end{array}$ & $\begin{array}{l}4.9998 \\
4.9983 \\
5.9988 \\
6.9951 \\
3.9999\end{array}$ & $\begin{array}{l}0.5 \\
1.6 \\
1.0 \\
1.7 \\
0.5\end{array}$ & $\begin{array}{l}-42.3 \\
-41.4 \\
-49.6 \\
-50.9 \\
-51.2\end{array}$ & $\begin{array}{l}0.5 \\
1.3 \\
1.0 \\
1.7 \\
0.3\end{array}$ & $\begin{array}{l}517 \\
393 \\
361 \\
981 \\
368\end{array}$ & $\begin{array}{r}88 \\
54 \\
73 \\
214 \\
29\end{array}$ \\
\hline $\begin{array}{l}433 C \\
433 C \\
433 C \\
433 C \\
433 C\end{array}$ & $\begin{array}{l}38 \\
39 \\
40 \\
41 \\
44\end{array}$ & $\begin{array}{l}423.5 \\
425.4 \\
426.7 \\
427.5 \\
432.3\end{array}$ & $\begin{array}{l}425.4 \\
426.7 \\
427.5 \\
428.7 \\
436.0\end{array}$ & $\begin{array}{l}6 \\
4 \\
3 \\
3 \\
6\end{array}$ & $\begin{array}{l}(1) \\
(1) \\
(1) \\
(0) \\
(1)\end{array}$ & $\begin{array}{l}300 \\
300 \\
400 \\
300 \\
200\end{array}$ & $\begin{array}{l}-50.3 \\
-55.3 \\
-50.4 \\
-53.9 \\
-50.3\end{array}$ & $\begin{array}{l}31.0 \\
35.8 \\
31.1 \\
34.5 \\
31.1\end{array}$ & $\begin{array}{r}634.3 \\
1252.0 \\
663.8 \\
15092.7 \\
802.2\end{array}$ & $\begin{array}{l}5.9921 \\
3.9976 \\
2.9970 \\
2.9999 \\
5.9938\end{array}$ & $\begin{array}{l}2.7 \\
2.6 \\
4.8 \\
1.0 \\
2.4\end{array}$ & $\begin{array}{l}-50.2 \\
-55.2 \\
-50.3 \\
-53.9 \\
-50.3\end{array}$ & $\begin{array}{l}2.4 \\
1.9 \\
2.7 \\
0.6 \\
2.3\end{array}$ & $\begin{array}{l}294 \\
169 \\
336 \\
294 \\
175\end{array}$ & $\begin{array}{r}20 \\
31 \\
2 \\
23 \\
76\end{array}$ \\
\hline
\end{tabular}


TABLE 3 - Continued

\begin{tabular}{|c|c|c|c|c|c|c|c|c|c|c|c|c|c|c|c|}
\hline Hole & Flow & $\begin{array}{l}\text { Top } \\
\text { (m) }\end{array}$ & $\begin{array}{l}\text { Bottom } \\
\text { (m) }\end{array}$ & $N$ & $(T)$ & $\mathrm{AF}$ & $I$ & VGL & $k$ & $R$ & $\alpha 95$ & $\bar{I}$ & $S_{I}$ & $\begin{array}{l}\mathrm{MDF} \\
(\mathrm{Oe})\end{array}$ & $S_{M D F}$ \\
\hline $433 \mathrm{C}$ & 45 & 436.0 & 442.5 & 6 & (1) & 200 & -47.3 & 28.5 & 3119.5 & 5.9984 & 1.2 & -47.3 & 1.1 & 437 & 123 \\
\hline $433 \mathrm{C}$ & 46 & 442.5 & 444.9 & 4 & (0) & 200 & -9.3 & 4.7 & 241.3 & 3.9876 & 5.9 & -9.2 & 4.3 & 348 & 61 \\
\hline $433 \mathrm{C}$ & 47 & 444.9 & 450.7 & 5 & (0) & 300 & -5.4 & 2.7 & 438.6 & 4.9909 & 3.7 & -5.4 & 3.1 & 251 & 50 \\
\hline $433 \mathrm{C}$ & 48 & 450.7 & 458.1 & 7 & (2) & 200 & -44.1 & 25.9 & 773.8 & 6.9922 & 2.2 & -44.1 & 2.2 & 408 & 106 \\
\hline $433 \mathrm{C}$ & 49 & 458.1 & 463.4 & 5 & (0) & 200 & -45.0 & 26.6 & 1583.0 & 4.9975 & 1.9 & -45.0 & 1.6 & 342 & 64 \\
\hline $433 \mathrm{C}$ & $51 \mathrm{~A}$ & 463.9 & 466.5 & 3 & $(0)$ & 400 & -50.8 & 31.5 & 653.6 & 2.9969 & 4.8 & -50.7 & 2.8 & 479 & 17 \\
\hline $433 \mathrm{C}$ & $51 \mathrm{~B}$ & 466.5 & 476.6 & 5 & (1) & 200 & -12.0 & 6.1 & 1929.5 & 4.9979 & 1.7 & -12.0 & 1.5 & 298 & 9 \\
\hline $433 \mathrm{C}$ & 52 & 476.6 & 480.0 & 6 & (1) & 200 & -13.6 & 6.9 & 1004.0 & 5.9950 & 2.1 & -13.6 & 2.0 & 325 & 13 \\
\hline $433 \mathrm{C}$ & 53 & 480.0 & 481.9 & 4 & (1) & 200 & -25.8 & 13.6 & 719.7 & 3.9958 & 3.4 & -25.7 & 2.5 & 434 & 73 \\
\hline $433 \mathrm{C}$ & 54 & 481.9 & 489.1 & 7 & (2) & 200 & -33.9 & 18.6 & 1384.9 & 6.9957 & 1.6 & -33.9 & 1.7 & 301 & 5 \\
\hline $433 C$ & 56 & 490.1 & 499.2 & 5 & (1) & 300 & -42.5 & 24.6 & 301.0 & 4.9867 & 4.4 & -42.4 & 3.7 & 223 & 59 \\
\hline $433 \mathrm{C}$ & 58 & 500.0 & 504.0 & 6 & (1) & 200 & -47.2 & 28.4 & 628.0 & 5.9920 & 2.7 & -47.1 & 2.5 & 289 & 40 \\
\hline $433 \mathrm{C}$ & 59 & 504.0 & 510.5 & 6 & (1) & 300 & -45.0 & 26.6 & 1466.8 & 5.9966 & 1.7 & -45.0 & 1.6 & 309 & 62 \\
\hline $433 \mathrm{C}$ & 60 & 510.5 & 515.5 & 6 & (1) & 300 & -44.2 & 25.9 & 460.3 & 5.9891 & 3.1 & -44.1 & 3.0 & 211 & 72 \\
\hline $433 \mathrm{C}$ & 61 & 515.5 & 518.0 & 5 & (0) & 200 & -43.3 & 25.2 & 2869.6 & 4.9986 & 1.4 & -43.3 & 1.2 & 311 & 54 \\
\hline $433 \mathrm{C}$ & 62 & 518.0 & 523.0 & 6 & (1) & 400 & -40.6 & 23.2 & 322.9 & 5.9845 & 3.7 & -40.5 & 3.4 & 368 & 23 \\
\hline $433 \mathrm{C}$ & 63 & 523.0 & 529.7 & 6 & (1) & 200 & -40.0 & 22.8 & 5617.5 & 5.9991 & 0.9 & -40.0 & 0.8 & 328 & 28 \\
\hline $433 \mathrm{C}$ & 64 & 529.7 & 539.4 & 6 & (1) & 400 & -68.1 & 51.2 & 143.7 & 5.9652 & 5.6 & -67.7 & 5.6 & 322 & 85 \\
\hline $433 \mathrm{C}$ & 65 & 539.4 & 545.3 & 5 & (0) & 400 & -66.1 & 48.5 & 3150.6 & 4.9987 & 1.4 & -66.1 & 1.2 & 488 & 73 \\
\hline $433 \mathrm{C}$ & 66 & 545.3 & 548.6 & 8 & (2) & 400 & -65.4 & 47.5 & 2561.8 & 7.9973 & 1.1 & -65.4 & 1.2 & 453 & 75 \\
\hline
\end{tabular}

Notes: Flow, flow unit number: SS indicates volcaniclastic sandstone layer. Top, Bottom: sub-bottom depths of flow top and bottom. $N, T$ : number of all the paleomagnetic samples and thermally demagnetized samples used for the calculation of mean inclination. AF: peak alternating field at which mean inclination is calculated (Oe). $I$, VGL: mean inclination and corresponding virtual geomagnetic latitude (degrees). $k$ : best estimate of Fisher's precision parameter. $R$ : best estimate of the resultant length of $N$ unit vectors. $\alpha 95$ : semi-angle of cone of confidence for $I$ at 95 per cent level (degrees). $I, S_{1}$ : arithmetic mean and standard deviation of inclination. $M D F$ : $S_{M D F}$ : mean and standard deviation of median demagnetizing field.

Inclination (deg)

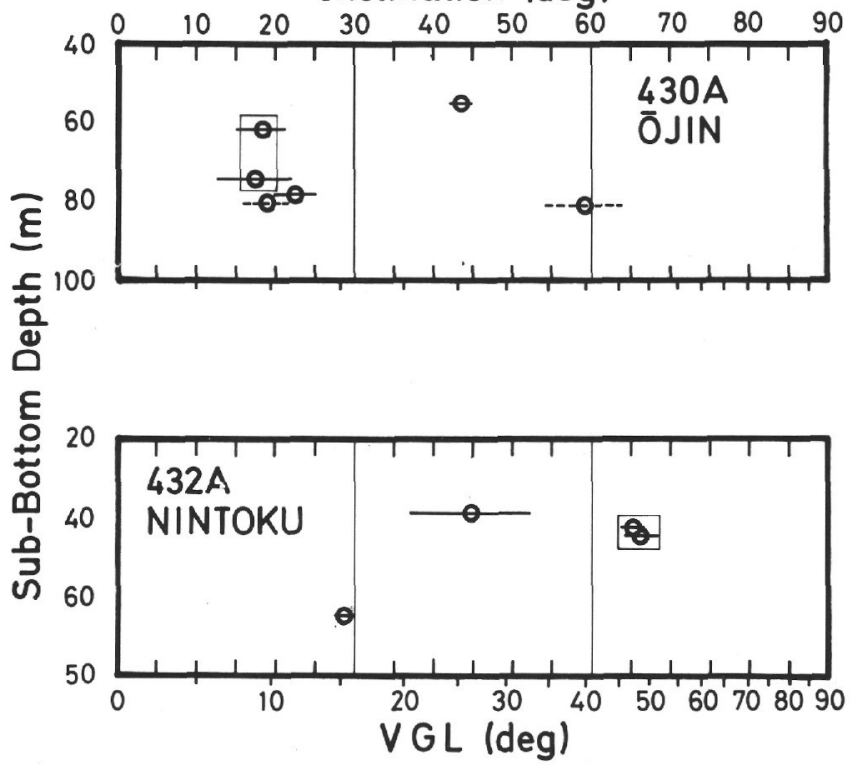

Figure 5. Flow mean inclination against depth at Ōjin and Nintoku. Error bars indicate $\alpha_{95}$ calculated by the method in Appendix A. Flow Units 4 and 5 at $\bar{O}$ jin have too few samples, so that $\alpha_{95}$ could not be determined. Boxes indicate flow units combined to form groups (Table 4).

lyzed magnetic profiles by stacking magnetic anomalies in the North Pacific, and found six short polarity events in the time interval of 54 to $70 \mathrm{~m} . y$. ago. The addition of these new events reduced the mean length of a polarity interval in that period from $0.85 \mathrm{~m} . \mathrm{y}$. (Heirtzler et al.) to $0.51 \mathrm{~m} . \mathrm{y}$. Assuming that the eruption of lavas is stochastic, described by the Poisson process, it is possible to estimate the time span covered by the successive lava flows, using the number of geomagnetic reversals recorded in the succession (Kono, 1973). In the case of Suiko, no magnetic reversals are recorded among 65 lava flows. Application of the statistical method of Kono (1973) yields an upper limit of $1.5 \mathrm{~m} . \mathrm{y}$. for these lavas at the 95 per cent confidence level (the mean length of a polarity interval is assumed to be $0.5 \mathrm{~m} . \mathrm{y}$.). It is therefore very unlikely that these flow units cover a time span of more than $1.5 \times 10^{6}$ years. Radiometric age of $64.7 \pm 1.1 \mathrm{~m}$.y. for Suiko (Dalrymple et al., this volume) is also in good agreement with this conclusion. It can also be pointed out that if all of the flow units erupted in a single reversed polarity interval, the maximum possible time span is the longest single reversed interval at about $65 \mathrm{~m} . \mathrm{y}$. ago, which is $0.64 \mathrm{~m} . \mathrm{y}$. (63-67 m.y.) or 1.93 m.y. (60-70 m.y.), according to a standard time scale (LaBrecque et al., 1977; corrected for new decay constants by Mankinen and Dalrymple, 1979). A time interval of more than $10^{4}$ years but less than 1.5 m.y. is a very convenient one to sample the paleosecular variation of the geomagnetic field; $10^{4}$ years is regarded as minimum interval to average out the secular variation, while in less than $10^{6}$ years we can neglect the disturbing effects of plate motions, geomagnetic polarity reversals, or excursions.

The fact that the PSV is really adequately sampled in these flows can also be shown by statistical methods. In Figure 7, mean inclination $(I)$ and its confidence limit $\left(\alpha_{95}\right)$ are shown for various partial assemblages of flow 


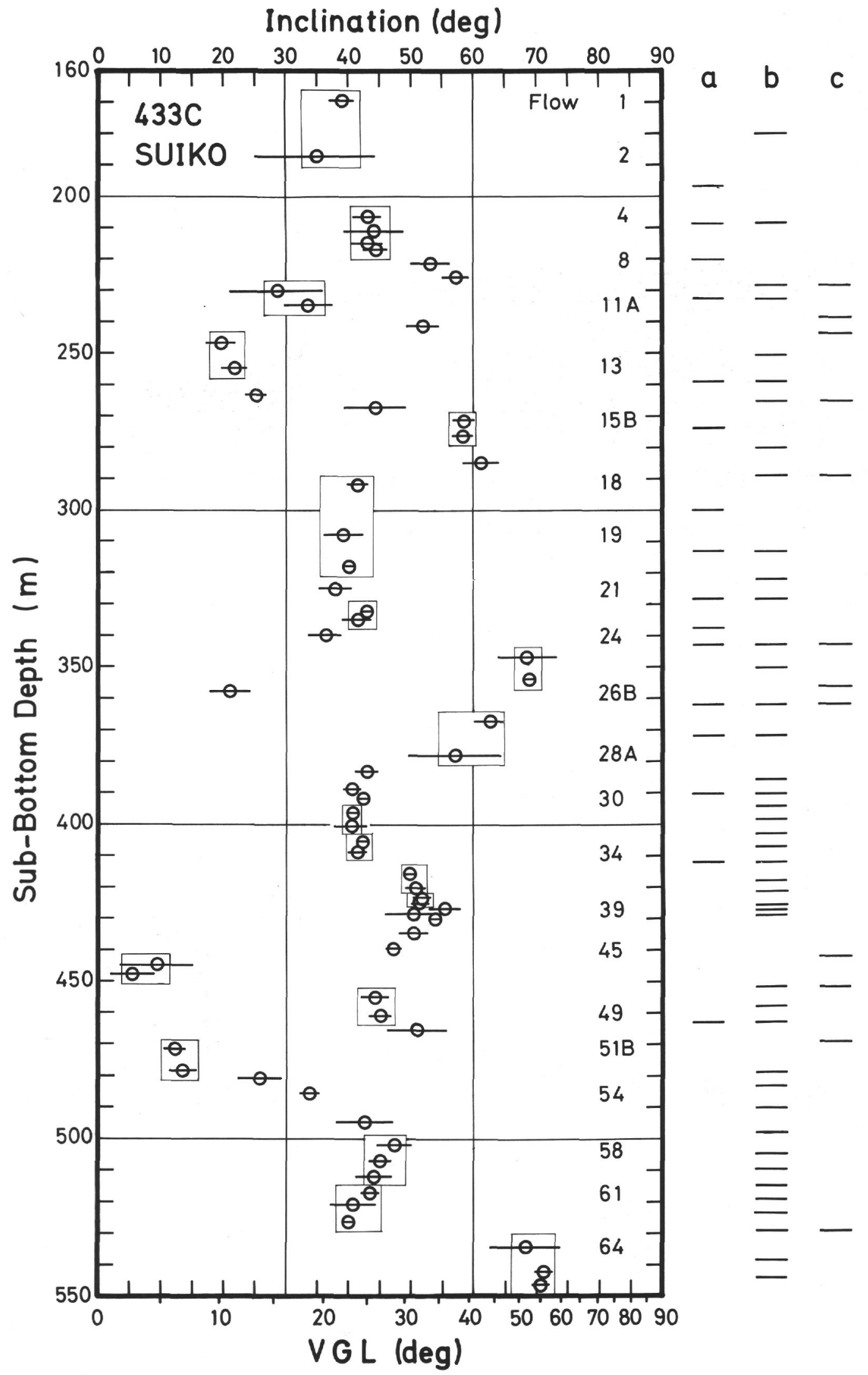

Figure 6. Flow mean inclination against depth at Suiko. The bars at right indicate possible time gaps suggested from changes in chemistry (a), flow-top erosion or oxidation (b), and jump in inclination of more than $15^{\circ}(\mathrm{c})$. For other explanations, see the legend of Figure 5. 
TABLE 4

Group Mean Inclination, Virtual Latitude, and Other Statistical Data

\begin{tabular}{|c|c|c|c|c|c|c|c|c|c|c|c|c|c|}
\hline Hole & Group & $N$ & $I$ & VGL & $k$ & $R$ & $\alpha 95$ & $I$ & $s_{1}$ & \multicolumn{4}{|c|}{$\begin{array}{l}\text { Flow Units } \\
\text { Included }\end{array}$} \\
\hline \multicolumn{14}{|c|}{ Ojin } \\
\hline $\begin{array}{l}430 A \\
430 A \\
430 A \\
430 A \\
430 A\end{array}$ & $\begin{array}{l}1 \\
2 \\
3 \\
4 \\
5\end{array}$ & $\begin{array}{r}4 \\
16 \\
6 \\
2 \\
1\end{array}$ & $\begin{array}{r}-43.2 \\
-17.7 \\
-22.4 \\
-18.8 \\
59.0\end{array}$ & $\begin{array}{r}25.2 \\
9.1 \\
11.7 \\
9.6 \\
39.8\end{array}$ & $\begin{array}{r}4852.9 \\
185.0 \\
583.3 \\
2.0 \\
2.0\end{array}$ & $\begin{array}{r}3.9994 \\
15.9189 \\
5.9914 \\
2.0000 \\
1.0000\end{array}$ & $\begin{array}{l}1.3 \\
2.7 \\
2.8 \\
2.0 \\
2.0\end{array}$ & $\begin{array}{r}-43.2 \\
-17.7 \\
-22.4 \\
-18.8 \\
59.0\end{array}$ & $\begin{array}{l}0.9 \\
4.3 \\
2.6 \\
0.2 \\
2.0\end{array}$ & $\begin{array}{c}\text { SS } \\
1 \\
3 \\
4 \\
5\end{array}$ & 2 & & \\
\hline \multicolumn{14}{|c|}{ Nintoku } \\
\hline $\begin{array}{l}432 \mathrm{~A} \\
432 \mathrm{~A} \\
432 \mathrm{~A}\end{array}$ & $\begin{array}{l}1 \\
2 \\
3\end{array}$ & $\begin{array}{r}3 \\
13 \\
14\end{array}$ & $\begin{array}{l}-44.6 \\
-66.0 \\
-28.6\end{array}$ & $\begin{array}{l}26.3 \\
48.3 \\
15.2\end{array}$ & $\begin{array}{r}263.6 \\
844.1 \\
1462.0\end{array}$ & $\begin{array}{r}2.9924 \\
12.9858 \\
13.9911\end{array}$ & $\begin{array}{l}7.6 \\
1.4 \\
1.0\end{array}$ & $\begin{array}{l}-44.5 \\
-65.9 \\
-28.6\end{array}$ & $\begin{array}{l}4.3 \\
2.1 \\
1.5\end{array}$ & $\begin{array}{c}\text { SS } \\
1 \\
3\end{array}$ & 2 & & \\
\hline \multicolumn{14}{|c|}{ Suiko } \\
\hline $\begin{array}{l}433 C \\
433 C \\
433 C \\
433 C \\
433 C\end{array}$ & $\begin{array}{l}1 \\
2 \\
3 \\
4 \\
5\end{array}$ & $\begin{array}{r}21 \\
31 \\
4 \\
5 \\
7\end{array}$ & $\begin{array}{l}-38.4 \\
-43.4 \\
-53.0 \\
-57.3 \\
-30.7\end{array}$ & $\begin{array}{l}21.6 \\
25.3 \\
33.6 \\
37.9 \\
16.5\end{array}$ & $\begin{array}{r}265.6 \\
300.9 \\
883.8 \\
1297.7 \\
164.0\end{array}$ & $\begin{array}{r}20.9247 \\
30.9003 \\
3.9966 \\
4.9969 \\
6.9634\end{array}$ & $\begin{array}{l}2.0 \\
1.5 \\
3.1 \\
2.1 \\
4.7\end{array}$ & $\begin{array}{l}-38.3 \\
-43.3 \\
-53.0 \\
-57.2 \\
-30.6\end{array}$ & $\begin{array}{l}3.6 \\
3.4 \\
2.2 \\
1.8 \\
4.8\end{array}$ & $\begin{array}{r}1 \\
4 \\
8 \\
9 \\
10\end{array}$ & $11 \mathrm{~A}$ & 6 & 7 \\
\hline $\begin{array}{l}433 C \\
433 C \\
433 C \\
433 C \\
433 C\end{array}$ & $\begin{array}{r}6 \\
7 \\
8 \\
9 \\
10\end{array}$ & $\begin{array}{r}6 \\
13 \\
8 \\
6 \\
9\end{array}$ & $\begin{array}{l}-52.0 \\
-20.8 \\
-25.3 \\
-44.3 \\
-58.4\end{array}$ & $\begin{array}{l}32.6 \\
10.8 \\
13.3 \\
26.0 \\
39.1\end{array}$ & $\begin{array}{r}684.3 \\
578.7 \\
1081.6 \\
184.3 \\
2268.9\end{array}$ & $\begin{array}{r}5.9927 \\
12.9793 \\
7.9935 \\
5.9729 \\
8.9965\end{array}$ & $\begin{array}{l}2.6 \\
1.7 \\
1.7 \\
4.9 \\
1.1\end{array}$ & $\begin{array}{l}-52.0 \\
-20.8 \\
-25.3 \\
-44.1 \\
-58.4\end{array}$ & $\begin{array}{l}2.3 \\
2.5 \\
1.9 \\
4.6 \\
1.3\end{array}$ & $\begin{array}{l}11 \mathrm{~B} \\
12 \\
14 \\
15 \mathrm{~A} \\
15 \mathrm{~B}\end{array}$ & 13 & & \\
\hline $\begin{array}{l}433 C \\
433 C \\
433 C \\
433 C \\
433 C\end{array}$ & $\begin{array}{l}11 \\
12 \\
13 \\
14 \\
15\end{array}$ & $\begin{array}{r}8 \\
22 \\
6 \\
8 \\
9\end{array}$ & $\begin{array}{l}-61.2 \\
-40.1 \\
-38.1 \\
-42.4 \\
-36.2\end{array}$ & $\begin{array}{l}42.3 \\
22.9 \\
21.4 \\
24.6 \\
20.1\end{array}$ & $\begin{array}{r}369.6 \\
545.5 \\
889.2 \\
2378.7 \\
382.3\end{array}$ & $\begin{array}{r}7.9811 \\
21.9615 \\
5.9944 \\
7.9971 \\
8.9791\end{array}$ & $\begin{array}{l}2.9 \\
1.3 \\
2.2 \\
1.1 \\
2.6\end{array}$ & $\begin{array}{l}-61.1 \\
-40.1 \\
-38.1 \\
-42.4 \\
-36.1\end{array}$ & $\begin{array}{l}3.2 \\
2.5 \\
2.1 \\
1.2 \\
3.1\end{array}$ & $\begin{array}{l}17 \\
18 \\
21 \\
22 \\
24\end{array}$ & $\begin{array}{l}19 \\
23\end{array}$ & 20 & \\
\hline $\begin{array}{l}433 C \\
433 C \\
433 C \\
433 C \\
433 C\end{array}$ & $\begin{array}{l}16 \\
17 \\
18 \\
19 \\
20\end{array}$ & $\begin{array}{l}9 \\
4 \\
9 \\
5 \\
5\end{array}$ & $\begin{array}{l}-687 \\
-21.0 \\
-60.2 \\
-43.0 \\
-40.4\end{array}$ & $\begin{array}{l}52.1 \\
10.9 \\
41.2 \\
25.0 \\
23.1\end{array}$ & $\begin{array}{r}757.6 \\
832.5 \\
146.1 \\
1622.6 \\
2627.2\end{array}$ & $\begin{array}{l}8.9894 \\
3.9964 \\
8.9452 \\
4.9975 \\
4.9985\end{array}$ & $\begin{array}{l}1.9 \\
3.2 \\
4.3 \\
1.9 \\
1.5\end{array}$ & $\begin{array}{l}-68.6 \\
-21.0 \\
-59.9 \\
-43.0 \\
-40.4\end{array}$ & $\begin{array}{l}2.0 \\
2.3 \\
4.8 \\
1.6 \\
1.3\end{array}$ & $\begin{array}{l}25 \\
26 \mathrm{~B} \\
27 \\
28 \mathrm{~B} \\
29\end{array}$ & $28 \mathrm{~A}$ & & \\
\hline $\begin{array}{l}433 C \\
433 C \\
433 C \\
433 C \\
433 C\end{array}$ & $\begin{array}{l}21 \\
22 \\
23 \\
24 \\
25\end{array}$ & $\begin{array}{r}5 \\
9 \\
10 \\
13 \\
10\end{array}$ & $\begin{array}{l}-42.3 \\
-40.5 \\
-41.9 \\
-50.3 \\
-50.7\end{array}$ & $\begin{array}{l}24.4 \\
23.1 \\
24.1 \\
31.1 \\
31.4\end{array}$ & $\begin{array}{r}4314.2 \\
1352.5 \\
3240.6 \\
1499.8 \\
948.6\end{array}$ & $\begin{array}{r}4.9991 \\
8.9941 \\
9.9972 \\
12.9920 \\
9.9905\end{array}$ & $\begin{array}{l}1.2 \\
1.4 \\
0.8 \\
1.1 \\
1.6\end{array}$ & $\begin{array}{l}-42.3 \\
-40.5 \\
-41.9 \\
-50.3 \\
-50.6\end{array}$ & $\begin{array}{l}1.0 \\
1.7 \\
1.1 \\
1.5 \\
1.9\end{array}$ & $\begin{array}{l}30 \\
31 \\
33 \\
35 \\
37\end{array}$ & $\begin{array}{l}32 \\
34 \\
36 \\
38\end{array}$ & & \\
\hline $\begin{array}{l}433 C \\
433 C \\
433 C \\
433 C \\
433 C\end{array}$ & $\begin{array}{l}26 \\
27 \\
28 \\
29 \\
30\end{array}$ & $\begin{array}{l}4 \\
3 \\
3 \\
6 \\
6\end{array}$ & $\begin{array}{l}-55.3 \\
-50.4 \\
-53.9 \\
-50.3 \\
-47.3\end{array}$ & $\begin{array}{l}35.8 \\
31.1 \\
34.5 \\
31.1 \\
28.5\end{array}$ & $\begin{array}{r}1252.0 \\
663.8 \\
15092.7 \\
802.2 \\
3119.5\end{array}$ & $\begin{array}{l}3.9976 \\
2.9970 \\
2.9999 \\
5.9938 \\
5.9984\end{array}$ & $\begin{array}{l}2.6 \\
4.8 \\
1.0 \\
2.4 \\
1.2\end{array}$ & $\begin{array}{l}-55.2 \\
-50.3 \\
-53.9 \\
-50.3 \\
-47.3\end{array}$ & $\begin{array}{l}1.9 \\
2.7 \\
0.6 \\
2.3 \\
1.1\end{array}$ & $\begin{array}{l}39 \\
40 \\
41 \\
44 \\
45\end{array}$ & & & \\
\hline $\begin{array}{l}433 C \\
433 C \\
433 C \\
433 C \\
433 C\end{array}$ & $\begin{array}{l}31 \\
32 \\
33 \\
34 \\
35\end{array}$ & $\begin{array}{r}9 \\
12 \\
3 \\
11 \\
4\end{array}$ & $\begin{array}{r}-7.1 \\
-44.5 \\
-50.8 \\
-12.9 \\
-25.8\end{array}$ & $\begin{array}{r}3.6 \\
26.2 \\
31.5 \\
6.5 \\
13.6\end{array}$ & $\begin{array}{r}237.9 \\
922.4 \\
653.6 \\
1051.8 \\
719.7\end{array}$ & $\begin{array}{r}8.9664 \\
11.9881 \\
2.9969 \\
10.9905 \\
3.9958\end{array}$ & $\begin{array}{l}3.3 \\
1.4 \\
4.8 \\
1.4 \\
3.4\end{array}$ & $\begin{array}{l}-7.1 \\
-44.5 \\
-50.7 \\
-12.9 \\
-25.7\end{array}$ & $\begin{array}{l}4.0 \\
2.0 \\
2.8 \\
1.9 \\
2.5\end{array}$ & $\begin{array}{l}46 \\
48 \\
51 \mathrm{~A} \\
51 \mathrm{~B} \\
53\end{array}$ & $\begin{array}{l}47 \\
49\end{array}$ & & \\
\hline $\begin{array}{l}433 C \\
433 C \\
433 C \\
433 C \\
433 C\end{array}$ & $\begin{array}{l}36 \\
37 \\
38 \\
39 \\
40\end{array}$ & $\begin{array}{r}7 \\
5 \\
18 \\
17 \\
19\end{array}$ & $\begin{array}{l}-33.9 \\
-42.5 \\
-45.5 \\
-41.2 \\
-66.4\end{array}$ & $\begin{array}{l}18.6 \\
24.6 \\
26.9 \\
23.6 \\
48.8\end{array}$ & $\begin{array}{r}1384.9 \\
301.0 \\
505.8 \\
541.4 \\
418.5\end{array}$ & $\begin{array}{r}6.9957 \\
4.9867 \\
17.9664 \\
16.9704 \\
18.9570\end{array}$ & $\begin{array}{l}1.6 \\
4.4 \\
1.5 \\
1.5 \\
1.6\end{array}$ & $\begin{array}{l}-33.9 \\
-42.4 \\
-45.4 \\
-41.2 \\
-66.3\end{array}$ & $\begin{array}{l}1.7 \\
3.7 \\
2.7 \\
2.5 \\
3.3\end{array}$ & $\begin{array}{l}54 \\
56 \\
58 \\
61 \\
64\end{array}$ & $\begin{array}{l}59 \\
62 \\
65\end{array}$ & $\begin{array}{l}60 \\
63 \\
66\end{array}$ & \\
\hline
\end{tabular}

Note: See Table 2 for explanation.

units at Site 433. The figure shows that, for larger, $N, I$ and $\alpha_{95}$ of partial sample do not differ statistically from those obtained from the whole units; i.e., these numbers remain essentially unchanged above about $N=30$.

Figures 8 and 9 show histograms of VGL obtained from this study, as well as from Midway and Meiji. The overall distribution of VGL for Suiko is quite unchanged when groups defined in the last section are taken as the unit of sample instead of flow units, again suggesting the adequacy of sampling of PSV.

Table 5 lists the summary of inclination statistics. As the number of data are small for Sites 430 and 432, the 


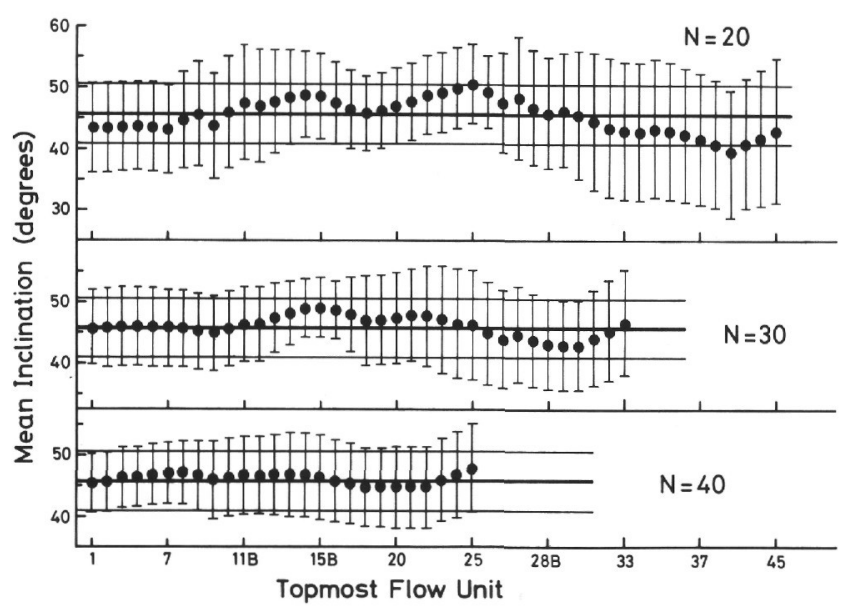

Figure 7. Estimates of mean inclinations and their 95 per cent confidence intervals $\left(\alpha_{95}\right)$ when only $\mathrm{N}$ consective flow units on Suiko are used as samples. Thick and thin horizontal lines indicate the mean inclination and $\alpha_{95}$ for all the flow units $(\mathrm{N}=65$, $\left.\mathrm{I}=45.7 \pm 4.8^{\circ}\right)$. When the number of samples is small $(\mathrm{N}=20)$, the statistical parameters do not always approximate the whole distribution well. When $\mathrm{N}$ is 30 or larger, the mean and $\alpha_{95}$ are not much different from those of all the flow units, which indicates that we have a statistically stable ensemble when $\mathrm{N} \geq 30$.

estimated mean inclinations have large errors. For Site 433 (Suiko), the data determine both the mean inclination and secular variation quite well. The table also includes statistics for parts of Suiko basalts. The fact that all the statistical parameters $\left(I, \alpha_{95}, k, s\right.$, etc.) corresponding to various sampling levels (all the flow units, groups, or parts of flow units) are quite similar indicates that the basalt sequence on Suiko forms a statistically stable ensemble. This again confirms that the PSV is adequately and sufficiently sampled in the inclination variation on Suiko. The paleolatitude $\phi$ was calculated by the dipole formula (1). The error angles in paleolatitude $(d \phi)$ and in inclination ( $d I$, written as $\alpha_{95}$ in this table) are related as

$$
\begin{aligned}
d \phi & =2 d I /\left(1+3 \cos ^{2} I\right) \\
& =1 / 2\left(1+3 \sin ^{2} \phi\right) d I .
\end{aligned}
$$

The parameter $s$ is the dispersion of field directions about the mean direction, and is calculated from the precision parameter $k$ as

$$
s^{2}=2 / k
$$

and $s_{u}$ and $s_{1}$ are 95 per cent confidence limits for $s$ (Cox, 1969). It can be concluded that the mean inclination for Suiko basalts is $45.7^{\circ} \pm 4.8^{\circ}$ at the 95 per cent confidence level. This value is significantly different from both the geocentric dipole field inclination of $63.3^{\circ}$ at the present latitude $\left(44.8^{\circ} \mathrm{N}\right)$ and that of $35.5^{\circ}$ at the latitude of Kilauea $\left(19.5^{\circ} \mathrm{N}\right)$.

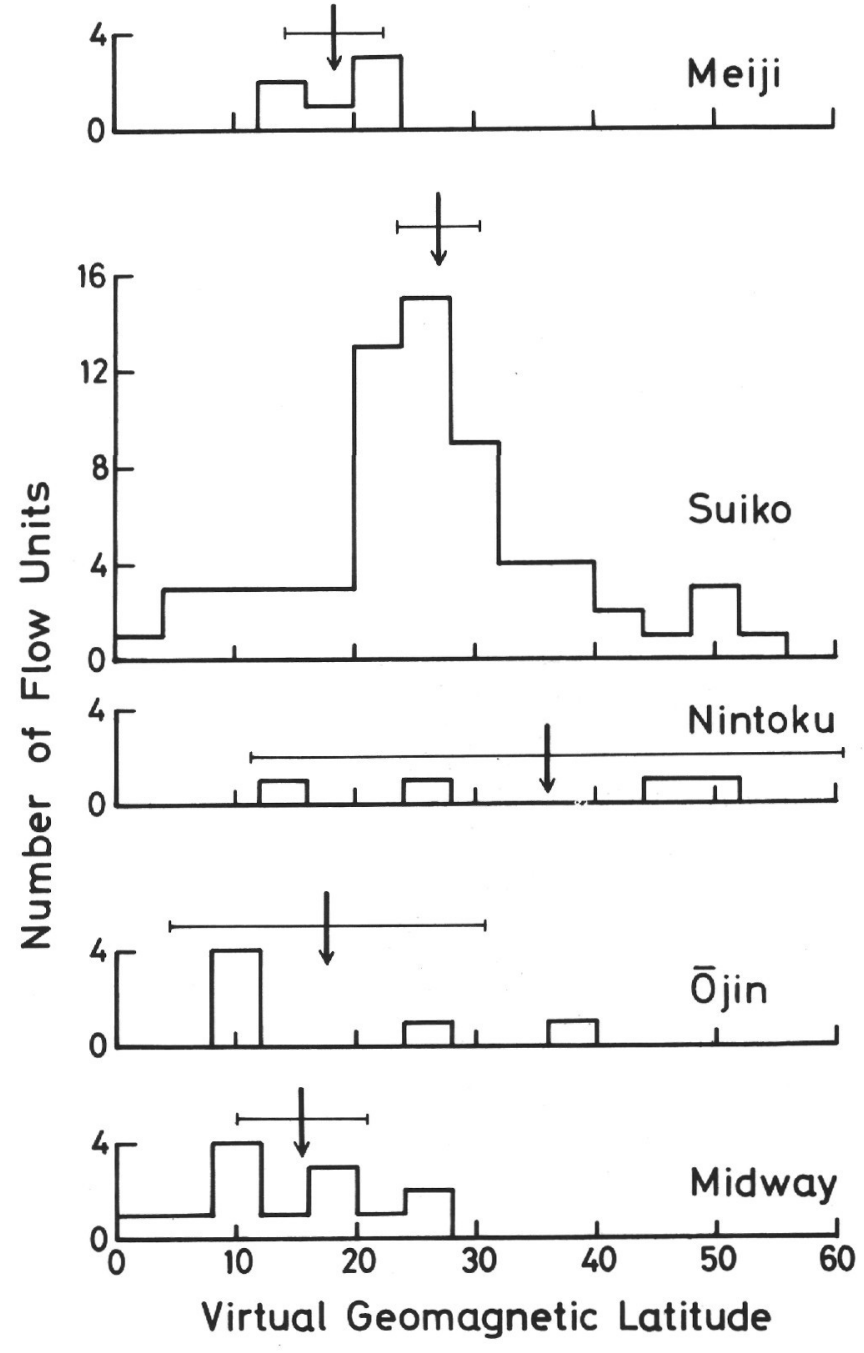

Figure 8. Histograms of flow mean VGL for some Emperor seamounts and Midway. Paleolatitudes and its 95 per cent confidence intervals are indicated by arrows and error bars.

Table 6 summarizes the statistics data of VGL. In this table, $K$ is the precision parameter of the distribution of virtual geomagnetic poles (VGPs) calculated assuming a Fisherian distribution of VGPs around the mean, and $S$ is the dispersion of VGPs obtained by

$$
S^{2}=2 / K \text {. }
$$

$S_{u}, S_{1}$ are 95 per cent confidence intervals determined by the method of Cox (1969). The mean VGL for Suiko basalts is much the same when different methods are used or when different samples are taken (Table 5 and 6 ). We shall use the value determined for magnetic groups $\left(26.9^{\circ} \pm 3.5^{\circ}\right)$ as the best estimate of the paleolatitude of Suiko. This again is significantly different from both the present latitude and the latitude of Kilauea.

Secular variations calculated from the Emperor Seamounts are much larger than the ones determined for 


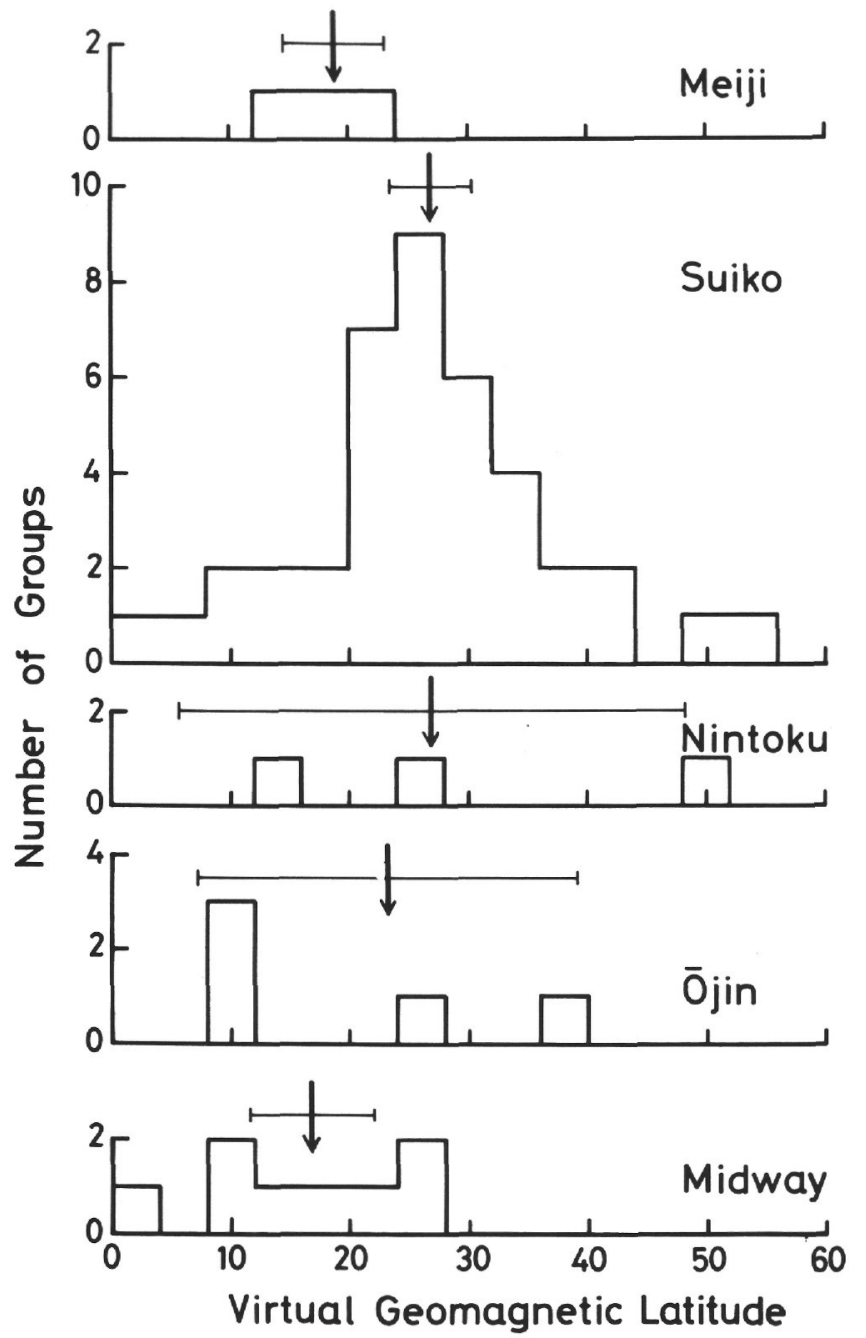

Figure 9. Histograms of group mean VGL. historic and Brunhes-age lavas on Hawaiian islands (Doell and Cox, 1971, 1972), but are similar to that determined for ${ }^{14} \mathrm{C}$-dated lavas on the island of Hawaii (Coe et al., 1978). As discussed earlier, the lavas on Suiko sample the PSV adequately and sufficiently. The possibility that some of the Hawaiian data show too small dispersions, owing to insufficient sampling of PSV, has been suggested by Coe et al. (1978). Although the confidence intervals are very large because the number of data is small, dispersions at Sites 430 (Ōjin) and 432 (Nintoku) are also consistent with larger secular variation. Table 7 lists the PSV data from some of the volcanoes originated on the Hawaiian hot spot. The paleolatitude and VGP dispersion data are also plotted against age in Figure 10. The table suggests that PSV may not be appropriately sampled in Midway and Meiji, mostly because the number of lava flows is too small, meaning that the paleolatitudes (VGL) calculated for these places (Grommé and Vine, 1972; Marshall, 1978) may have much larger ambiguity than the nominal dispersions suggest.

\section{DISCUSSION AND CONCLUSIONS}

(1) The paleolatitude of Suiko Seamount is $27^{\circ}(\mathrm{N})$ with an uncertainty of $3.5^{\circ}$ at the 95 per cent confidence level. Since the sampling of PSV is adequate and since the magnetic stability is very high in this lava sequence, this estimate can be taken at its face value.

(2) The 400-meter basaltic section cored on top of Suiko Seamount seems, from magnetic data, to have accumulated in a period greater than $10^{4}$ years but less than $1.5 \times 10^{6}$ years. This estimate is consistent with the radiometric age data (Dalrymple et al., this volume). Some flow units seem to have erupted in quick succession, whereas time gaps appear between other flow units, suggesting that the volcanism was intermittent and

TABLE 5

Statistics of Inclination Data

\begin{tabular}{|c|c|c|c|c|c|c|c|c|c|c|}
\hline & $N$ & $I$ & $\alpha 95$ & $\bar{I}$ & S.D. & $k$ & $s$ & $s_{u}$ & $s_{1}$ & $\begin{array}{l}\text { Corresponding } \\
\text { latitudea }\end{array}$ \\
\hline \multicolumn{11}{|c|}{ Flow units as individual data } \\
\hline $\bar{O}$ jin $\quad(430)$ & 6 & -30.6 & 17.8 & -29.8 & 17.3 & 15.2 & 20.8 & 32.8 & 15.2 & $16.5 \pm 11.0$ \\
\hline Nintoku (432) & 4 & -55.9 & 29.2 & -51.2 & 18.1 & 10.9 & 24.5 & 43.1 & 17.2 & $36.4 \pm 30.1$ \\
\hline Suiko (433) & 65 & -45.7 & 4.8 & -43.2 & 14.0 & 14.5 & 21.3 & 24.0 & 17.8 & $27.1 \pm 3.9$ \\
\hline Suiko, top 30 flows ${ }^{b}$ & 30 & -45.6 & 6.4 & -43.9 & 13.6 & 17.9 & 19.2 & 23.2 & 16.3 & $27.0 \pm 5.2$ \\
\hline Suiko, middle 30 flows ${ }^{\mathrm{c}}$ & 30 & -47.9 & 6.1 & -45.9 & 12.3 & 19.4 & 18.4 & 22.3 & 15.7 & $29.0 \pm 5.2$ \\
\hline Suiko, bottom 30 flows ${ }^{d}$ & 30 & -46.6 & 8.6 & -42.7 & 15.8 & 10.4 & 25.1 & 30.5 & 21.4 & $27.9 \pm 7.1$ \\
\hline \multicolumn{11}{|c|}{ Flow groups as individual data } \\
\hline Site 430 & 5 & -33.4 & 21.5 & -32.2 & 18.2 & 13.6 & 22.0 & 36.3 & 15.8 & $18.2 \pm 13.9$ \\
\hline Site 432 & 3 & -48.7 & 34.1 & -46.4 & 18.8 & 14.1 & 21.6 & - & - & $29.6 \pm 29.6$ \\
\hline Site 433 & 40 & -45.5 & 5.9 & -43.2 & 13.6 & 15.6 & 20.5 & 24.2 & 17.8 & $27.0 \pm 4.8$ \\
\hline
\end{tabular}

Notes: All the angles in degrees. $N$, number of samples; $I, \alpha 95$, mean inclination and its 95 per cent confidence limit by Fisherian statistics (see Appendix A); $\bar{I}$, S.D., arithmetic mean and standard deviation of inclinations; $k$, precision parameter of inclination distribution; $s, s_{\mathcal{U}}, s_{1}$, angular standard deviation and its 95 per cent confidence limits (Cox, 1969).

${ }_{\mathrm{b}}^{\mathrm{a}}$ Calculated from $I$ and $\alpha 95$ by eq. (3).

Includes Flow Units 433-1 through 433-28B.

Includes Flow Units 433-17 through 46.

$\mathrm{d}_{\text {Includes Flow Units 433-33 through 433-66. }}$ 
TABLE 6

Statistics of Virtual Geomagnetic Latitude Data

\begin{tabular}{|c|c|c|c|c|c|c|c|c|c|c|c|}
\hline $\begin{array}{l}\text { Seamount } \\
\text { Name }\end{array}$ & Site & $N$ & Lat. & $\alpha_{95}$ & $\overline{\mathrm{VGL}}$ & S.D. & $k$ & $s$ & $s_{\mathcal{u}}$ & $s_{1}$ & $\begin{array}{l}\text { Corresponding } \\
\text { Inclination }^{\mathrm{a}}\end{array}$ \\
\hline \multicolumn{12}{|c|}{ Flow units as individual data } \\
\hline$\overline{\mathrm{O}} \mathrm{jin}$ & $(430)$ & 6 & 17.6 & 13.2 & 17.4 & 12.6 & 26.6 & 15.7 & 24.8 & 11.5 & $-32.5 \pm 20.7$ \\
\hline Nintoku & $(432)$ & 4 & 36.0 & 24.6 & 34.4 & 16.4 & 15.0 & 20.9 & 36.7 & 14.6 & $-55.5 \pm 24.2$ \\
\hline Suiko & (433) & 65 & 27.1 & 3.4 & 26.6 & 11.1 & 28.0 & 15.3 & 17.4 & 13.7 & $-45.7 \pm 4.2$ \\
\hline Midwayb & & 13 & 15.4 & 5.4 & 15.3 & 7.6 & 61.0 & 10.3 & 14.0 & 8.2 & $28.9 \pm 8.9$ \\
\hline Meiji & $(192)^{\mathrm{c}}$ & 6 & 19.2 & 4.1 & 19.2 & 3.8 & 264.1 & 5.0 & 7.9 & 3.6 & $34.8 \pm 6.2$ \\
\hline \multicolumn{12}{|c|}{ Flow groups as individual data } \\
\hline$\overline{\mathrm{O} j \mathrm{jin}}$ & $(430)$ & 5 & 23.2 & 16.0 & 22.5 & 14.6 & 18.5 & 18.8 & 31.1 & 13.5 & $-40.5 \pm 21.8$ \\
\hline Nintoku & (432) & 3 & 26.9 & 21.3 & 26.3 & 15.6 & 19.6 & 18.3 & - & - & $-45.4 \pm 26.4$ \\
\hline Suiko & (433) & 40 & 26.9 & 3.5 & 26.3 & 11.3 & 26.0 & 15.9 & 18.8 & 13.8 & $-45.4 \pm 4.3$ \\
\hline Midwayb & & 8 & 16.8 & 5.2 & 16.7 & 7.3 & 65.7 & 10.0 & 14.8 & 7.6 & $31.2 \pm 8.3$ \\
\hline Meiji & $(192)^{\mathrm{c}}$ & 3 & 18.9 & 4.2 & 18.9 & 4.0 & 250.6 & 5.1 & - & - & $34.5 \pm 6.4$ \\
\hline
\end{tabular}

Notes: All angles in degrees. $N$, number of samples; Lat., $\alpha 95$, mean VGL and its 95 per cent confidence limit by Fisherian statistics (see Appendix A); VGL, S.D., arithmetic mean and standard deviation of VGL; $k$, precision parameter of VGP distribution; $s, s_{U}, s_{1}$, angular standard deviation and its 95 per cent confidence limits of VGP distribution (Cox, 1969).

${ }_{b}^{\mathrm{a}}$ Calculated from lat. and $\alpha 95$ by eq. (3).

Data from Grommé and Vine (1972).

${ }^{\mathrm{c}}$ Data from Marshall (1978).

TABLE 7

Some of the Paleosecular Variation Data on Hawaiian Hot Spot

\begin{tabular}{|c|c|c|c|c|c|c|c|c|}
\hline \multirow{3}{*}{$\begin{array}{c}\text { Volcano or } \\
\text { Volcanic Series }\end{array}$} & \multirow[b]{3}{*}{ Agea } & \multirow{3}{*}{$\begin{array}{l}\text { Present } \\
\text { Latitude }\end{array}$} & \multicolumn{4}{|c|}{$\begin{array}{c}\text { Paleomagnetic } \\
\text { Data }\end{array}$} & \multirow{2}{*}{\multicolumn{2}{|c|}{$\begin{array}{c}\text { Angular } \\
\text { Dispersion }\end{array}$}} \\
\hline & & & & Paleo- & & VGP & & \\
\hline & & & $N$ & latitude & $\alpha 95$ & $s$ & $s_{u}$ & $s_{1}$ \\
\hline Hawaii (Historic) ${ }^{b}$ & 1750-1960 A.D. & 19.5 & 17 & 19.9 & 1.4 & 3.3 & 4.3 & 2.6 \\
\hline Hawaii ( ${ }^{14} \mathrm{C}$ dated $) \mathrm{b}$ & $2000-18000$ y.B.P. & 19.5 & 8 & 17.8 & 10.7 & 15.5 & 24.4 & 11.4 \\
\hline Hawaii (Selected Brunhes) b,c & $0.01-0.7$ & 19.5 & 129 & 16.3 & 1.9 & 12.7 & 13.9 & 11.7 \\
\hline Oahu (Koolau) & $1.8-2.6$ & 21.4 & 33 & 16.9 & 3.6 & 11.4 & 13.7 & 9.8 \\
\hline Oahu (Waianae) ${ }^{\mathrm{d}}$ & $2.4-3.6$ & 21.5 & 55 & 15.7 & 3.3 & 13.6 & 15.7 & 11.9 \\
\hline Kauai (Makaweli)e & $3.5-4.0$ & 22.0 & 24 & 15.6 & 3.1 & 8.4 & 10.4 & 7.0 \\
\hline Kauai (Napali)e & $4.5-5.6$ & 22.1 & 46 & 14.9 & 3.1 & 11.9 & 13.9 & 10.4 \\
\hline Nihoaf & $7.0 \pm 0.3$ & 23.1 & 14 & 21.0 & 6.6 & 13.5 & 17.9 & 10.8 \\
\hline Midwayg & $27.0 \pm 0.6$ & 28.2 & 13 & 15.4 & 5.4 & 10.3 & 14.0 & 7.6 \\
\hline Ōjin & $55.2 \pm 0.7$ & 38.0 & 6 & 17.6 & 13.2 & 15.7 & 24.8 & 11.5 \\
\hline Nintoku & $56.2 \pm 0.6$ & 41.3 & 4 & 36.0 & 24.6 & 20.9 & 36.7 & 14.6 \\
\hline Suiko & $64.7 \pm 1.1$ & 44.8 & 65 & 27.1 & 3.4 & 15.3 & 17.4 & 13.7 \\
\hline Meijih & $69-72$ & 53.0 & 6 & 19.2 & 4.1 & 5.0 & 7.9 & 3.6 \\
\hline
\end{tabular}

Notes: Angles in degrees.

${ }_{\mathrm{b}}^{\mathrm{A}} \mathrm{Age}$ in million years unless otherwise stated.

${ }^{\mathrm{b}}$ Coe et al. (1978).

Includes Kiekie volcanic series on Niihau and Honolulu volcanic series on Oahu.

doell and Dalrymple (1973).

e Doell (1972a).

f Doell (1972b).

g Grommé and Vine (1972).

$\mathrm{h}_{\text {Marshall (1978). }}$

episodic, just as in the volcanoes of the island of Hawaii. The time interval estimated above also suggests that a substantial part of a seamount may have formed within a relatively short time, within a polarity interval, say. This explains why some of the seamount magnetic anomalies can be interpreted using a simple model (uniform magnetization), and gives some support to the paleomagnetism of seamount magnetic anomalies (e.g., Harrison et al., 1975).
(3) A good record of paleosecular variation in inclination about $65 \mathrm{~m}$.y. ago was obtained. The Suiko data (Figure 6) contain some portions where almost continuous changes are recorded, 12 or more time gaps, and about 15 extreme (minimum and maximum) values. They cover a time span long enough to sample the entire range of secular variation but short enough so that we can neglect the disturbing effects of geomagnetic polarity transition or plate motions. A precision parameter of 


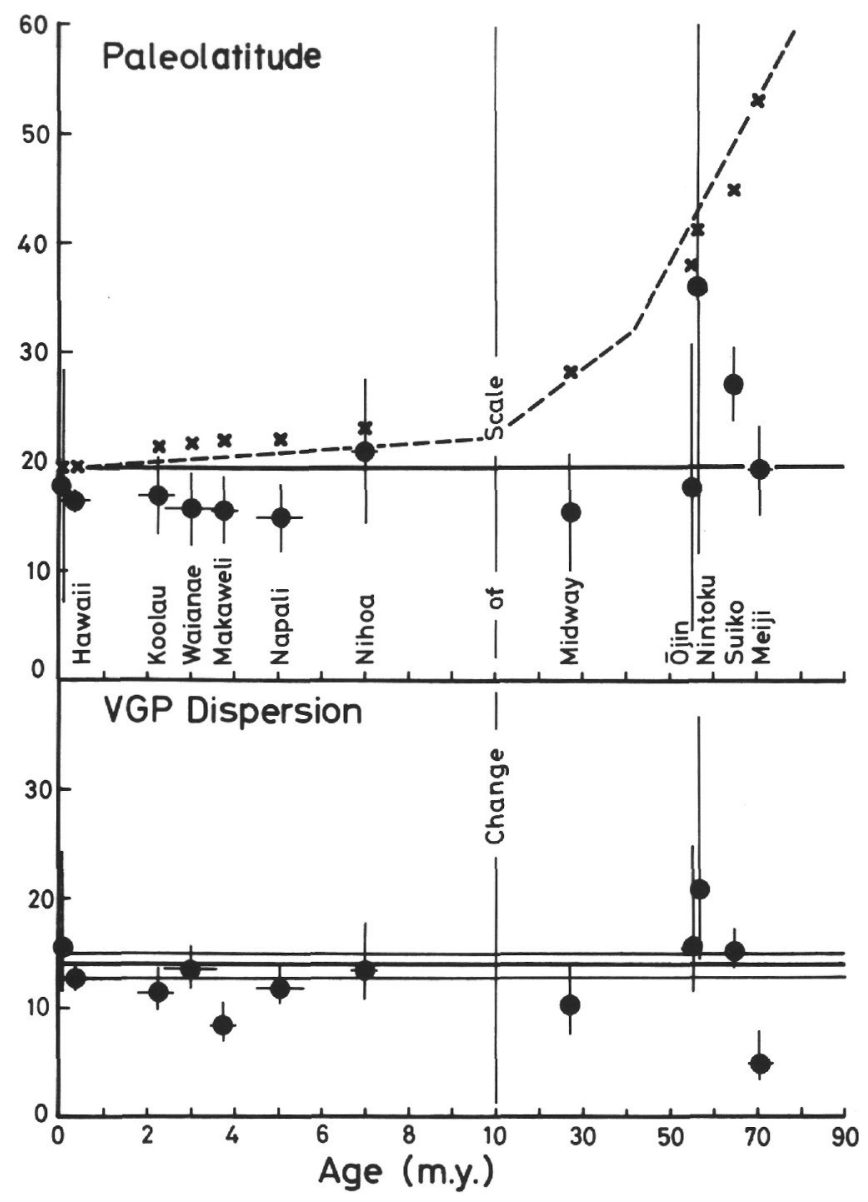

Figure 10. Paleolatitudes and VGP dispersions of some selected volcanoes of Hawaiian hot spot. Horizontal bars indicate duration of volcanic activity or errors in dating. "Hawaii"' are ${ }^{14} \mathrm{C}$-dated and selected Brunhes epoch data of Coe et al. (1978). Vertical bars are $\alpha_{95}$ for paleolatitude and 95 per cent confidence limits $\left(\mathrm{S}_{\mathrm{u}}, \mathrm{S}_{1}\right)$ of VGP dispersion $\mathrm{S}$. Crosses show the present locality of the volcanoes and dashed line marks the trace of Hawaiian hot spot for the constant speed of the Pacific plate movement at $8 \mathrm{~cm} /$ year. Most of VGP dispersion data are compatible with $\mathrm{S}=$ $14^{\circ} \pm 1^{\circ}$ (horizontal lines). Note the somewhat low dispersion on Midway and extremely low dispersion on Meiji.

$K=26$ was obtained from the distribution of VGL, which corresponds to a VGP dispersion $(S)$ of $15.9^{\circ}$. This dispersion is significantly larger than those of historical or Brunhes-age lavas on Hawaiian islands (Doell and Cox, 1971, 1972), but is similar to that of ${ }^{14} \mathrm{C}$-dated flows on the island of Hawaii (Coe et al., 1978). It is also consistent with PSV models C and D of Cox (1970), which predict VGP dispersions of $14.3^{\circ}$ and $14.6^{\circ}$, respectively, at the latitude of $27^{\circ}$. This suggests that the magnitude of PSV at the Hawaiian hot spot either is similar, through the period 0 to $70 \mathrm{~m} . \mathrm{y}$. ago, to the worldwide trend, if adequate sampling is done (which is the conclusion of Coe et al., 1978), or has changed significantly between 65 and 1 m.y. ago. If the secular variation at the Hawaiian hot spot was indeed similar to the world average (at least for 25-70 m.y.), then the paleomagnetic data from Midway $(27.7 \pm 0.6$ m.y., Dalrymple et al., 1977) and Meiji (69-72 m.y.) may represent insufficient sampling of PSV.

\section{ACKNOWLEDGMENTS}

I am grateful to many of the technical staff on board the Glomar Challenger for help in sampling and maintenance of instruments, and especially to Toshiaki Takayama for preparation and bookkeeping of samples. I thank Toshiyuki Tosha for thermal demagnetization measurements.

\section{REFERENCES}

Blakely, R. and Cox, A., 1972. Evidence for short geomagnetic polarity intervals in the early Cenozoic, J. Geophys. Res., v. 77, pp. 7065-7072.

Briden, J. C. and Ward, M. A., 1966. Analysis of magnetic inclinations in borecores, Pure Appl. Geophys., v. 63, pp. 133-152.

Carnahan, B., Luther, H. A., and Wilkes, J. O., 1969. Applied Numerical Methods. New York (John Wiley), p. 542.

Coe, R. S., Grommé, S., and Mankinen, E. A., 1978. Geomagnetic paleointensities from radiocarbon-dated lava flows on Hawaii and the question of the Pacific nondipole low, $J$. Geophys. Res., v. 83, pp. 1740-1756.

Cox, A., 1969. Confidence limits for the precision parameter k, Geophys. J., v. 18, p. 545-549.

1970. Latitude dependence of the angular dispersion of the geomagnetic field, Geophys. J., v. 20, pp. 253-269.

Crow, E. L., Davis, F. A., and Maxfield, M. W., 1960. Statistics Manual. New York (Dover), pp. 53-54.

Dalrymple, G. B., Clague, D. A., and Lanphere, M. A., 1977. Revised age for Midway Volcano, Hawaiian Volcanic chain, Earth Planet. Sci. Lett., v. 37, pp. 107-116.

Doell, R. R. and Cox, A., 1971. Pacific geomagnetic secular variation, Science, v. 171 , pp. 248-254.

1972. The Pacific geomagnetic secular variation anomaly and the question of lateral uniformity in the lower mantle. In Robertson, E. C. (Ed.), The Nature of the Solid Earth: New York (McGraw-Hill), pp. 245-284.

Fisher, R., 1953. Dispersion on a sphere, Proc. Roy. Soc. Lond., Ser. A, v. 217, pp. 295-305.

Grommé, S. and Vine, F. J., 1972. Paleomagnetism of Midway Atoll lavas and northward movement of the Pacific plate, Earth Planet. Sci. Lett., v. 17, pp. 159-168.

Harrison, C. G. A., Jarrard, R. D., Vacquier, V., and Larson, R. L., 1975. Paleomagnetism of Cretaceous Pacific seamounts, Geophys. J., v. 42, pp. 859-882.

Heirtzler, J. R., Dickson, G. O., Herron, E. M., Pitman, W. C. III, and Le Pichon, X., 1968. Marine magnetic anomalies, geomagnetic field reversals, and motions of the ocean floor and continents, J. Geophys. Res., v. 73, pp. 21192136.

Kono, M., 1973. Geomagnetic polarity changes and the duration of volcanism in successive lava flows, J. Geophys. Res., v. 78, pp. 5972-5982.

La Brecque, J. L., Kent, D. V., and Cande, S. C., 1977. Revised magnetic polarity time scale for late Cretaceous and Cenozoic time, Geology, v. 5, pp. 330-335.

Mankinen, E. A. and Dalrymple, G. B., 1979. Revised geomagnetic polarity time scale for the interval 0 to $5 \mathrm{~m} . \mathrm{y}$. B.P., J. Geophys. Res., v. 83.

Marshall, M., 1978. The magnetic properties of some DSDP basalts from the North Pacific and inferences for Pacific plate tectonics, J. Geophys. Res., v. 83, pp. 289-308. 
Morgan, W. J., 1971. Convection plumes in the lower mantle, Nature, v. 230, pp. 42-43.

1972. Plate motions and deep mantle convection, Geol. Soc. Am. Mem., v. 132, pp. 7-22.

Wilson, J. T., 1963a. A possible origin of the Hawaiian Islands, Can. J. Phys., v. 41, pp. 863-870.

$1963 \mathrm{~b}$. Evidence from islands on the spreading of the ocean floor, Nature, v. 197, pp. 536-538.

\section{APPENDIX: STATISTICS OF INCLINATION DATA}

A statistical method which can be used when only inclinations are known was developed by Briden and Ward (1966). They started from the widely accepted assumption in paleomagnetism that the distribution of NRM directions about the true mean direction has the form proposed by Fisher (1953). The frequency density is then

$$
f\left(\theta^{\prime}, \psi^{\prime}\right) d \theta^{\prime} d \psi^{\prime}=\frac{\kappa}{4 \pi \sinh \kappa} e^{\kappa \cos \theta^{\prime}} \sin \theta^{\prime} d \theta^{\prime} d \psi^{\prime},
$$

where $\theta^{\prime}$ and $\psi^{\prime}$ are the angular displacement from the true mean direction and azimuthal angle, and $\kappa$ is the precision parameter describing how well the distribution is concentrated near the true mean. Although $\theta^{\prime}$ and $\psi^{\prime}$ form polar coordinates with respect to the true mean direction, it is more convenient to express (A1) in a coordinate system measured from the vertical. If we denote the new coordinate variables by $\theta$ and $\psi$, then

$$
\begin{aligned}
& \cos \theta^{\prime}=\cos \theta_{0} \cos \theta+\sin \theta_{0} \sin \theta \cos \psi \\
& \text { and } \\
& \qquad \sin \theta^{\prime} d \theta^{\prime} d \psi^{\prime}=\sin \theta d \theta d \psi,
\end{aligned}
$$

where $\theta_{0}$ is the angular distance from the true mean direction to the vertical. It follows from (Al) that the frequency density in the new coordinates is

$$
\begin{aligned}
f(\theta, \psi)= & \frac{\kappa}{4 \pi \sinh \kappa} \exp \left[\kappa \left(\cos \theta_{0} \cos \theta\right.\right. \\
& \left.\left.+\sin \theta_{0} \sin \theta \cos \psi\right)\right] \sin \theta
\end{aligned}
$$

Noting that

$$
\begin{aligned}
\frac{1}{2 \pi} \int_{0}^{2 \pi} e^{x \cos \psi} d \psi & =\frac{1}{2 \pi} \sum_{n=0}^{\infty} \frac{x^{n}}{n !} \int_{0}^{2 \pi} \cos ^{n} \psi d \psi \\
& =\sum_{n=0}^{\infty} \frac{x^{2 n}}{2^{2 n}(n !)^{2}} \\
& \equiv I_{0}(x) \quad \text {....Modified Bessel function. }
\end{aligned}
$$

Briden and Ward obtained the marginal distribution $f(\theta)$ from (A2),

$$
\begin{aligned}
f(\theta) & =\int_{0}^{2 \pi} f(\theta, \psi) d \psi \\
& =\frac{\kappa}{2 \sinh \kappa} e^{\kappa \cos \theta_{0} \cos \theta} I_{0}\left(\kappa \sin \theta_{0} \sin \theta\right) \sin \theta .
\end{aligned}
$$

The problem now is to find some means to estimate both $\theta_{0}$ and $\kappa$ from observed data. They chose expectations of $\sin \theta$ and $\cos \theta$ for this purpose:

$$
E(\cos \theta)=\int_{0}^{\pi} \cos \theta f(\theta) d \theta
$$

$$
\begin{aligned}
= & \frac{\kappa}{\sinh \kappa} \sum_{m=0}^{\infty} \sum_{n=0}^{\infty} \frac{\left(\kappa \cos \theta_{0}\right)^{2 m+1}\left(\kappa \sin \theta_{0}\right)^{2 n}}{(2 m+1) ! 2^{2 n}(n !)^{2}} \\
& \times \frac{(2 m+1) ! !(2 n) ! !}{(2 m+2 n+3) ! !}
\end{aligned}
$$

$$
\begin{aligned}
E(\sin \theta)= & \frac{\pi \kappa}{2 \sinh \kappa} \sum_{m=0}^{\infty} \sum_{n=0}^{\infty} \frac{\left(\kappa \cos \theta_{0}\right)^{2 m}\left(\kappa \sin \theta_{0}\right)^{2 n}}{(2 m) ! 2^{2 n}(n !)^{2}} \\
& \times \frac{(2 m-1) ! !(2 n+1) ! !}{(2 m+2 n+2) ! !}
\end{aligned}
$$

As they could not express (A4) and (A5) by elementary functions, they integrated them numerically and produced tables and a figure for the functions.

$$
\begin{aligned}
& \Lambda^{*}\left(\kappa, \theta_{0}\right)=1 /\left[1-\sqrt{E(\cos \theta)^{2}+E(\sin \theta)^{2}}\right], \\
& \text { and } \\
& \Theta^{*}\left(\kappa, \theta_{0}\right)=\tan ^{-1}[E(\sin \theta) / E(\cos \theta)] .
\end{aligned}
$$

Their method of estimating $\theta_{0}$ and $\kappa$ was to calculate

$$
\begin{aligned}
& \Lambda=1 /\left[1-\sqrt{\left.\bar{c}^{2}+\bar{s}^{2}\right]}\right] \\
& \Theta=\tan ^{-1}(\bar{s} / \bar{c})
\end{aligned}
$$

where $\bar{c}$ and $\bar{s}$ are averages of observed cosines and sines

$$
\begin{aligned}
& \bar{c}=\frac{1}{N} \sum_{i=1}^{\mathrm{N}} \cos \theta_{i} \\
& \bar{s}=\frac{1}{N} \sum_{i=1}^{N} \sin \theta_{i},
\end{aligned}
$$

and to find the values of $\kappa$ and $\theta_{0}$ in the tables for which

$$
\begin{aligned}
& \Lambda^{*}\left(\kappa, \theta_{0}\right)=\Lambda, \\
& \Theta^{*}\left(\kappa, \theta_{0}\right)=\Theta .
\end{aligned}
$$

However, as can be seen from their paper, it is very difficult to prepare a table with such a widely varying parameter as $\kappa$ which takes values in the range $10^{0}$ to $10^{4}$ in usual paleomagnetic samples. Also, when $\kappa$ is small or when $\theta_{0}$ is large, interpolation in the tables produces large errors in their estimates. It is much better to have some analytical means to estimate $\theta_{0}$ and $\kappa$, following the distribution (A3).

Although Briden and Ward decided that (A4) and (A5) cannot be analytically integrated, (A4) can actually be integrated easily. In fact, expectations of any power of $\cos \theta$ or any even power of $\sin \theta$ or their products can be integrated. I shall only treat the integration of $E\left(\cos ^{n} \theta\right)$ in this paper. The moment-generating function of $\cos \theta \operatorname{can}$ be derived from the original distribution function $f(\theta)$ as (Carnahan et al., 1969)

$$
\begin{aligned}
M_{\cos \theta}(t) & =E\left(e^{t \cos \theta}\right)=\int_{0}^{\pi} e^{t \cos \theta} f(\theta) d \theta \\
& =\frac{\kappa}{2 \sinh \kappa} \int_{0}^{\pi} e^{t \cos \theta} e^{\kappa \cos \theta} 0 \cos \theta \sum_{n=0}^{\infty} \frac{\left(\kappa \sin \theta_{0} \sin \theta\right)^{2 n}}{2^{2 n}(n !)^{2}} \sin \theta d \theta
\end{aligned}
$$




$$
\begin{aligned}
= & \frac{\kappa}{2 \sinh \kappa} \sum_{n=0}^{\infty} \sum_{m=0}^{\infty} \frac{\left(\kappa \sin \theta_{0}\right)^{2 n}}{2^{2 n}(n !)^{2}} \frac{\left(\kappa \cos \theta_{0}+t\right)^{m}}{m !} \\
& \cdot \int_{0}^{\pi}(\sin \theta)^{2 n+1}(\cos \theta)^{m} d \theta .
\end{aligned}
$$

Since both $(\sin \theta)^{2 n+1}$ and $(\cos \theta)^{2 m}$ are symmetric about $\theta=\pi / 2$ and $(\cos \theta)^{2 m+1}$ antisymmetric, we obtain

$$
\begin{aligned}
M_{\cos \theta}(t)= & \frac{\kappa}{2 \sinh \kappa} \sum_{n=0}^{\infty} \sum_{m=0}^{\infty} \frac{\left(\kappa \sin \theta_{0}\right)^{2 n}\left(\kappa \cos \theta_{0}+t\right)^{2 m}}{2^{2 n}(n !)^{2}(2 m) !} \\
& \cdot 2 \int_{0}^{\pi / 2}(\sin \theta)^{2 n+1}(\cos \theta)^{2 m} d \theta \\
= & 2 \sinh \kappa \sum_{n=0}^{\infty} \sum_{m=0}^{\infty} \frac{\left(\kappa \sin \theta_{0}\right)^{2 n}\left(\kappa \cos \theta_{0}+t\right)^{2 m}}{2^{2 n}(n !)^{2}(2 m) !} \\
& \cdot \frac{2 \cdot(2 n) ! !(2 m-1) ! !}{(2 n+2 m+1) ! !} \\
= & \frac{\kappa}{\sinh \kappa} \sum_{n=0}^{\infty} \sum_{m=0}^{\infty} \frac{\left(\kappa \sin \theta_{0}\right)^{2 n}\left(\kappa \cos \theta_{0}+t\right)^{2 m}(n+m) !}{m ! n !(2 n+2 m+1) !}
\end{aligned}
$$

In the above reduction, we used the relation

$$
\begin{aligned}
& \int_{0}^{\pi / 2} \cos ^{2 m} x \sin ^{2 n+1} x d x=\frac{(2 m-1) ! !(2 n) ! !}{(2 m+2 n+1) ! !} \\
& (2 n) ! !=2^{n} n !, \quad(2 n+1) ! !=\frac{(2 n+1) !}{2^{n} n !}
\end{aligned}
$$

To sum the double infinite series, we replace $m+n$ by $n$; then the range of summation for $m$ is now from 0 to $n$ and

$$
\begin{aligned}
M_{\cos \theta}(t) & =\frac{\kappa}{\sinh \kappa} \sum_{n=0}^{\infty} \sum_{m=0}^{n} \frac{\left(\kappa \sin \theta_{0}\right)^{2 n-2 m}\left(\kappa \cos \theta_{0}+t\right)^{2 m} n !}{(2 n+1) ! m !(n-m) !} \\
& =\frac{\kappa}{\sinh \kappa} \sum_{n=0}^{\infty} \frac{\left(\kappa \sin \theta_{0}\right)^{2 n}}{(2 n+1) !}\left[1+\left(\frac{\kappa \cos \theta_{0}+t}{\kappa \sin \theta_{0}}\right)^{2}\right]^{n} \\
& =\frac{\kappa}{\sinh \kappa} \frac{\sinh \left[\kappa^{2}+2 \kappa t \cos \theta_{0}+t^{2}\right]}{\kappa^{2}+2 \kappa t \cos \theta_{0}+t^{2}}
\end{aligned}
$$

Thus, we have obtained the moment-generating function $M_{\cos \theta}(t)$, and we can calculate $n$th moment of $\cos \theta$ about the origin, from the relation

$$
\mu_{n^{\prime}}=E\left(\cos ^{n} \theta\right)=\int_{0}^{\pi} \cos ^{n} \theta f(\theta) d \theta
$$

$$
=\left[\frac{\partial^{n} M \cos \theta(t)}{\partial t^{n}}\right]_{t=0}
$$

In particular,

$$
E(\cos \theta)=\cos \theta_{0} L(\kappa),
$$

$$
E\left(\cos ^{2} \theta\right)=\cos ^{2} \theta_{0}+\frac{1-3 \cos ^{2} \theta_{0}}{\kappa} L(\kappa)
$$

where $L$ is the Langevin function

$$
L(x)=\operatorname{coth} x-\frac{1}{x}
$$

Unfortunately, $E(\cos \theta)$ cannot be expressed in an analytical form.

Since $M_{\cos \theta}(t)$ is a two-parameter function, the original distribution $f(\theta)$ can be completely determined by specifying any combination of two different moments $\mu_{n}^{\prime}$ and $\mu_{m}{ }^{\prime}$. I chose the first and second moments $E(\cos \theta)$ and $E\left(\cos ^{2} \theta\right)$, and equate them with the mean values derived from the observed inclinations. The best estimates of the mean inclination $\left(\mathbf{I}=\frac{\pi}{2}-\theta_{0}\right)$ and the precision parameter $(k)$ can be determined by solving the simultaneous equations

$$
\begin{aligned}
& \sin I\left(\operatorname{coth} k-\frac{1}{k}\right)=\frac{1}{N} \sum_{i=1}^{N} \sin I_{i} \\
& \sin ^{2} I+\frac{1-3 \sin ^{2} I}{k}\left(\operatorname{coth} k-\frac{1}{k}\right)=\frac{1}{N} \sum_{i=1}^{N} \sin ^{2} I_{i}
\end{aligned}
$$

where $\mathrm{I}_{i}(i=1,2, \ldots, N)$ are the observed inclinations $\left(I_{i}=\frac{\pi}{2}-\theta_{i}\right)$. The solutions of (A11) and (A12) can easily be obtained by iteration using a computer. When we calculate statistics of virtual geomagnetic longitude (VGL), we can simply replace inclinations by VGL in (A11) and (A12).

To estimate the uncertainties in $\theta_{0}$ and $\mathrm{K}$ determined from the inclination data, Briden and Ward (1966) proposed a method to calculate standard deviations $\sigma_{\theta_{0}}$ and $\sigma_{\kappa}$ by assuming nearly normal distribution of $c$ and $s$ about the mean $(\bar{c}, \bar{s})$ and also linear dependences of $c-\bar{c}$ and $s-\bar{s}$ on $\kappa-\bar{\kappa}$ and $\theta_{0}-\bar{\theta}_{0}$ (overbars denote observed or estimated values). However, these assumptions are not well satisfied (especially when $\theta_{0}$ or $\kappa$ is large), and the standard deviations are not good when we try to compare such uncertainties with those derived from the ordinary paleomagnetic data (where both inclination and declination are known). Actually, the best estimate of precision parameter $(k)$ and the cosine of angular error $(\delta)$ are related though the equations derived by Fisher (1953);

$$
\begin{gathered}
1-\delta=\frac{N-R}{R}\left\{\left(\frac{1}{P}\right)^{1 /(N-1)}-1\right\} \\
k=\frac{N-1}{N-R}
\end{gathered}
$$

where $P$ is probability that the cosine of error angle is less than $\delta$. We take $P=0.05$ and obtain the "best estimate" of the 95 per cent confidence limit

$$
\alpha_{95}=\cos ^{-1} \mid 1-\frac{N-1}{(k-1) N+1}\left\{20^{1 /(N-1)}-1 \mid\right] .
$$

Confidence limits for $k$ can be calculated by the method of Cox (1969). 